\title{
Kartierte Risikokonflikte als Instrument reflexiver Wissenspolitik
}
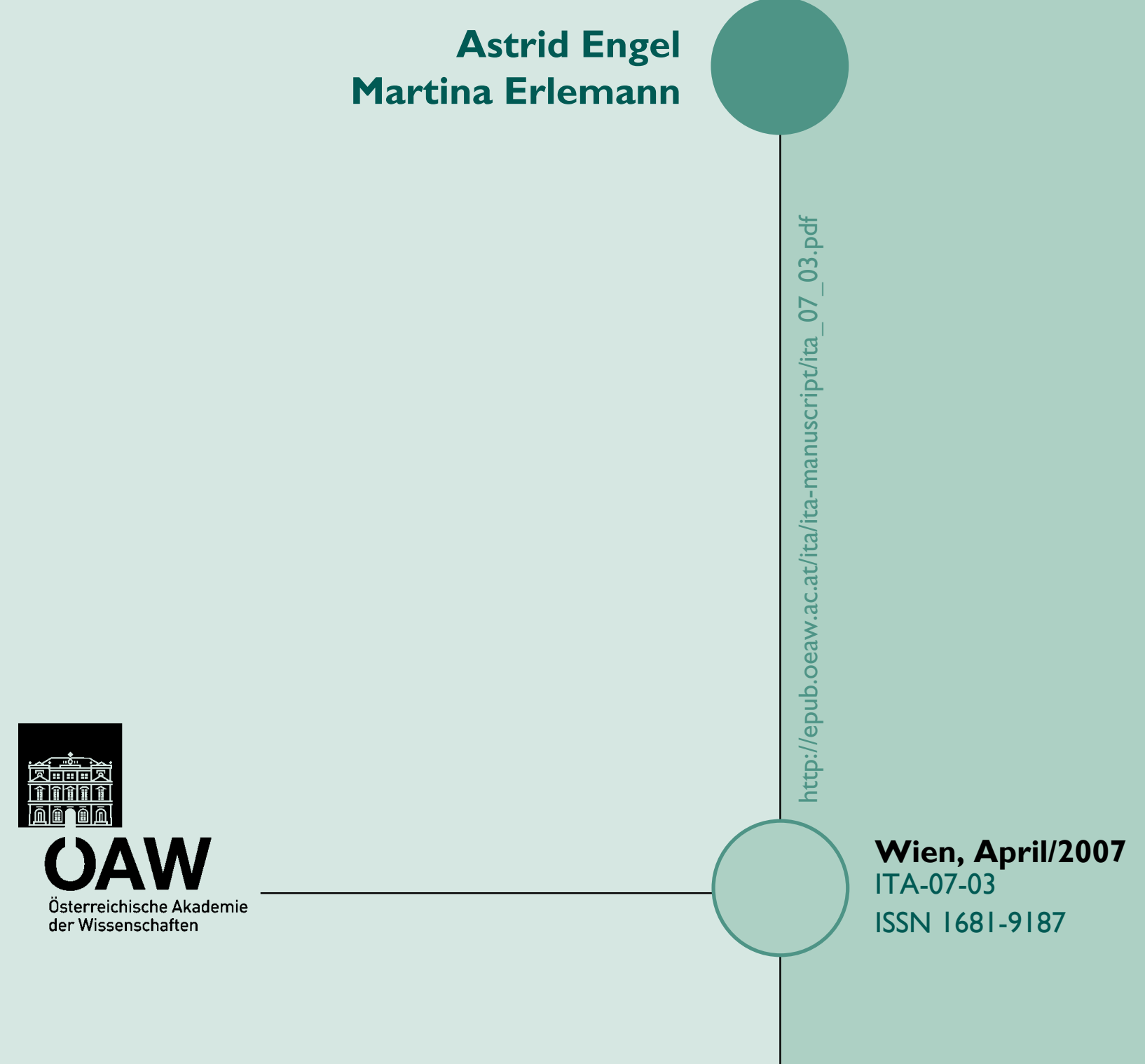



\title{
Kartierte Risikokonflikte als Instrument reflexiver Wissenspolitik
}

\section{Astrid Engel*), Martina Erlemann**)}

*) Münchener Projektgruppe für Sozialforschung e.V. (MPS)

**) Wissenschaftszentrum Umwelt (WZU), Universität Augsburg

\section{Keywords}

Visualisierung, Risikodiskurs, systemische Risiken, reflexive Wissenspolitik, Nahrungsergänzungsmittel, Nanotechnologie

\begin{abstract}
Die mit der Entwicklung neuer Technologien einher gehende neue Qualität von Risiken erfordert ein Überdenken der Strategien, die den gesellschaftlichen Umgang mit diesen Risiken betreffen. Die besondere systemische Qualität dieser Risiken führt zur Notwendigkeit einer veränderten Risikopolitik sowie Risikokommunikation. Basierend auf den Konzepten der systemischen Risiken und der reflexiven Wissenspolitik stellen wir eine so genannte „Risiko-Kartierung“ als ein neues, internetbasiertes Werkzeug für Risikokommunikation vor. Der im Rahmen des Projektes „Risikokonflikte kartiert" entwickelte Prototyp möchte mittels einer ungewöhnlichen methodischen Vorgehensweise bei der Analyse und visuellen Aufbereitung von Risikokonflikten einen veränderten gesellschaftlichen Umgang mit diesen anstoßen. Die Aufbereitung erfolgt exemplarisch anhand zweier Fallstudien, nämlich Nahrungsergänzungsmittel und nanoskalige Materialien.
\end{abstract}




\section{Inhalt}

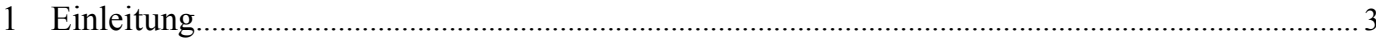

2 Der systemische Charakter zeitgenössischer Risiken ................................................................... 4

2.1 Der Risikobegriff in der Soziologie: Ausgewählte Aspekte ................................................................ 4

2.2 Der Begriff „,systemische Risiken“ im Projekt „Risikokonflikte kartiert“ .......................................... 6

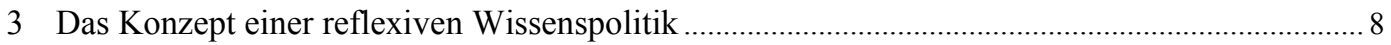

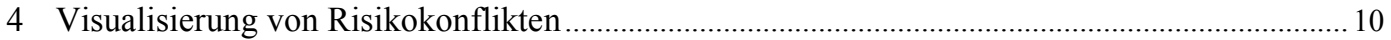

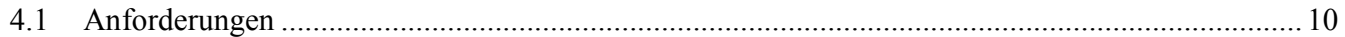

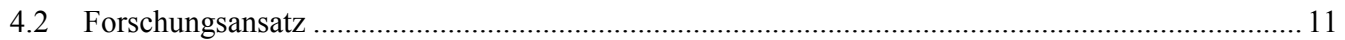

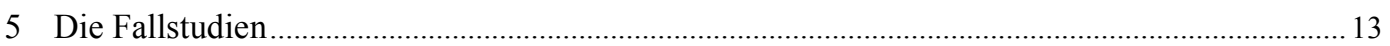

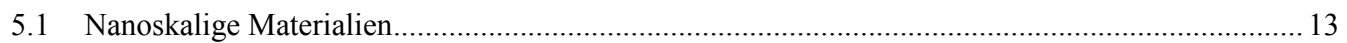

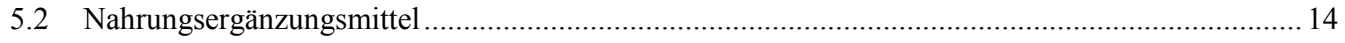

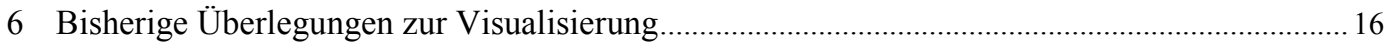

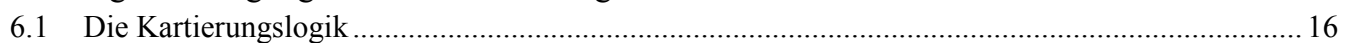

6.2 Strukturelemente der Kartierung am Beispiel Nahrungsergänzungsmittel ........................................ 18

6.3 Die Chronologie der Diskurse am Beispiel der Nanopartikel-Debatte ............................................ 19

7 Der Beitrag von kartierten Risikokonflikten zu Risikodebatten ..................................................... 22

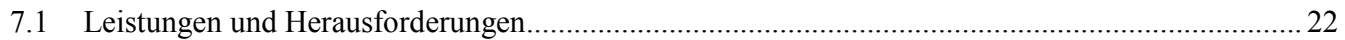

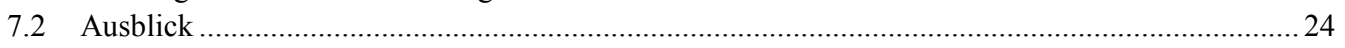

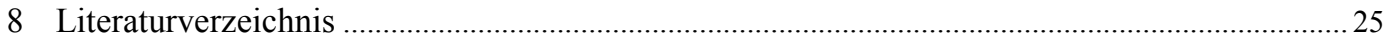

Dieser Beitrag basiert auf einem Vortrag im Rahmen der Sechsten Österreichischen TA-Konferenz, „TA'06. Vermessen, codiert, entschlüsselt? Potenziale und Risken der zunehmenden Datenverfügbarkeit“, Internationale Konferenz, Wien, 29. Mai 2006

\section{IMPRESSUM}

\section{Medieninhaber:}

Österreichische Akademie der Wissenschaften

Juristische Person öffentlichen Rechts (BGBI 569/|92I idF BGB| I |30/2003)

Dr. Ignaz Seipel-Platz 2, A-1010 Wien

\section{Herausgeber:}

Institut für Technikfolgen-Abschätzung (ITA)

Strohgasse 45/5, A- 1030 Wien

http://www.oeaw.ac.at/ita

Die ITA-manu:scripts erscheinen unregelmäßig und dienen der Veröffentlichung

von Arbeitspapieren und Vorträgen von Institutsangehörigen und Gästen.

Die manu:scripts werden ausschließlich über das Internetportal „epub.oeaw“

der Öffentlichkeit zur Verfügung gestellt:

http://epub.oeaw.ac.at/ita/ita-manuscript

ITA-manuscript Nr.: ITA-07-03 (April/2007)

ISSN-online: I 8I8-6556

http://epub.oeaw.ac.at/ita/ita-manuscript/ita_07_03.pdf

c 2007 ITA - Alle Rechte vorbehalten 


\section{Einleitung}

Die rezenten Technologieentwicklungen, wie etwa im Bereich der Bio-, Nano- oder auch der Kommunikationstechnologien, eröffnen eine Fülle von Anwendungsmöglichkeiten in ganz unterschiedlichen Bereichen, sei es in verschiedenen Industriebranchen, in der Medizin oder im Bereich der Konsumgüter. Nicht zuletzt die Vielfalt dieser Innovationen und deren oftmals komplexe Wirkungszusammenhänge machen die Folgen und Nebenfolgen dieser Technologien nahezu unüberschaubar. Jede Form des Risikomanagements und der Risikopolitik steht daher vor großen Herausforderungen, wenn es um Modelle einer partizipativen Technikfolgenabschätzung geht, die mit einem „empowerment“ zivilgesellschaftlicher Akteure einhergehen soll. Im Rahmen risikopolitischer Debatten wurde hier der Ruf nach „technological“ oder „scientific citizenship“ immer lauter. Die Frage nach seiner Umsetzung in den gesellschaftlichen Aushandlungsprozessen um die Gestaltung von Technologien wird denn auch nach wie vor lebhaft diskutiert. Insbesondere, wenn es um die Frage der Anwendung bzw. Nichtanwendung von Technologien geht, muss einer demokratischen Technikgestaltung (Abels und Bora 2004), die zivilgesellschaftliche Akteure in die Entscheidungsprozesse einbezieht, eine gelungene Risikokommunikation vorangehen.

Ein mögliches Instrument für eine Risikokommunikation, die einen veränderten gesellschaftlichen Umgang mit neuen Risiken anstoßen kann, stellt die Visualisierung von Risikodiskursen dar. Im Rahmen des Forschungsprojektes „Risikokonflikte visualisiert - Entwicklung und Erprobung von internetbasierten Argumentationslandkarten" ${ }^{1}$, das in diesem Beitrag vorgestellt wird, wird ein Prototyp für die Darstellung von Risikodiskursen in Form von internetbasierten Argumentationslandkarten entwickelt. Internetbasierung bedeutet dabei mehr als nur die Veröffentlichung unserer Forschungsergebnisse in Form einer Visualisierung, die sich Internet-NutzerInnen bei Interesse über eine Website anschauen können. Vielmehr wird ein Software-Tool entwickelt, das es den NutzerInnen ermöglicht, sich über eine interaktive Website einen Risikodiskurs nach eigenen Bedürfnissen und Interessen visualisieren zu lassen und über die interaktiven Elemente selbst an den Risikodebatten teilzunehmen. Dabei eröffnet das Tool einen neuen Blick auf die Komplexität und Unüberschaubarkeit technologischer Risiken, indem es in strukturierender Weise diese Komplexität wieder überschaubar macht und insbesondere zivilgesellschaftlichen Akteuren die Möglichkeit zur Teilnahme an risikopolitischen Diskursen bietet.

Der Artikel geht zunächst auf die Besonderheiten von systemischen Risiken ein und erläutert das Konzept einer reflexiven Wissenspolitik. Denn Risikopolitik, die sich im Spannungsfeld zwischen Wissenschaft, Politik und Öffentlichkeit bewegt, wirft Probleme auf, für deren Lösung es einer angemessenen Wissenspolitik bedarf. Anschließend wird das Vorhaben einer Kartierung von Risikodiskursen vorgestellt. Dabei diskutieren wir, was eine solche Visualisierung leisten kann und welche Herausforderungen ein solches Instrument mit sich bringt.

1 Das Projekt wird im Förderschwerpunkt Sozial-ökologische Forschung (SÖF) des Bundesministeriums für Bildung und Forschung (BMBF) zum Thema „Strategien zum Umgang mit systemischen Risiken“ vom Wissenschaftszentrum Umwelt (WZU) der Universität Augsburg und der Münchner Projektgruppe für Sozialforschung e.V. (MPS) unter Leitung von Stefan Böschen, Cordula Kropp und Jens Soentgen durchgeführt. Näheres hierzu siehe unter www.sozialforschung.org und unter

www.wzu.uni-augsburg.de/Projekte/Visualisierte_Risikokonflikte/Visualisierte_Risikokonflikte.html. 


\section{Der systemische Charakter zeitgenössischer Risiken}

\section{I Der Risikobegriff in der Soziologie: Ausgewählte Aspekte}

Mit der Entwicklung moderner Technologien und vor allem auch aufgrund der Erfahrung von Schäden infolge des Einsatzes dieser Technologien haben sich die gesellschaftliche Risikowahrnehmung und auch die Strategien zum Umgang mit ihnen deutlich gewandelt. „Risiko“ als Thema spätmoderner Gesellschaften hat sich - nicht zuletzt durch die „Katastrophenerfahrungen“ seit den 70er Jahren (Tschernobyl, Bhopal, Seveso, Harrisburg), die die bisher unbekannte zeitliche und räumliche Reichweite technisch-wissenschaftlicher Eingriffe in natürliche Kreisläufe deutlich werden ließen - immer stärker in den Vordergrund geschoben (Lau 1989). Lau spricht in diesem Kontext sogar von einem ,neuen Typus gesellschaftlicher Interessenkonflikte“ (ebd. 420), bei dem es nicht zuletzt um die Frage der Verteilung von Risikobetroffenheit und Risikokosten gehe. Beck, der den Begriff der „Risikogesellschaft“ (Beck 1986) geprägt hat, stellt das Problem des Risikos in den Kontext einer Theorie der Moderne. Er prognostiziert, dass die Probleme der Risikoverteilung wichtiger würden als die Konflikte der Reichtumsverteilung (ebd. 25). Die Debatte über die Anwendung neuer Technikentwicklungen und die damit verbundenen Risiken, die lange Zeit überwiegend in Expertenkreisen statt fand und von einer naturwissenschaftlich-technischen Sichtweise dominiert wurde, öffnet sich zunehmend für die gesamte Gesellschaft. Aufgrund des Wandels und der zunehmenden Komplexität von Ursachen und Wirkungen wird eine klare Definition von Risiken immer schwieriger. Daher spielt zunächst die Auseinandersetzung darüber, was eigentlich unter einem Risiko zu verstehen sei, eine große Rolle und, daraus folgend, welche Strategien des Umgangs mit den drohenden Schäden angemessen wären. Die Überzeugung setzt sich durch, dass die bisher üblichen wissenschaftlich-technologischen Methoden nicht ausreichten, sondern eine Betrachtung, die soziale und kulturelle Aspekte sowie Wechselwirkungen mit anderen Systemen mit einbezieht, notwendig wäre.

Die zunehmende gesellschaftliche Bedeutung der Debatte, wie mit Risiken umzugehen sei, wurde auch von der Soziologie aufgegriffen - dies nicht zuletzt aufgrund der Erkenntnis, dass die bisherige disziplinäre Begrenzung und die damit verbundene restriktive Verknüpfung von Risiko und Technik nicht ausreichten, um alle Risikodimensionen des Einsatzes neuer Technologien zu erfassen. Dabei wurden die soziologischen Arbeiten insbesondere in der Anfangszeit stark durch die Erfahrungen von Risiko und Unsicherheit infolge anscheinend unabwendbarer ökologischer Gefährdungen geprägt (Bonß 1995, 13).

Ein wesentlicher Beitrag der sozialwissenschaftlichen Forschung zur aktuellen Risikodiskussion besteht darin, die soziale und kulturelle Einbettung der Risikobewertung zu verdeutlichen. So führt beispielsweise Lau aus: „Was als Risiko begriffen wird und was nicht, welche Aspekte des Risikos wahrgenommen und untersucht werden und vor allem, wie die möglichen Gefahren zu werten sind, lässt sich [...] nicht ohne Rückgriff auf gesellschaftliche Wahrnehmungsmuster und Werte beantworten“ (Lau 1989, 419). Welches Risiko akzeptabel ist, welches nicht und welche Schlussfolgerungen für einen gesellschaftlichen Umgang daraus zu ziehen sind, hängt somit offensichtlich weniger von wissenschaftlichen Erkenntnissen als vielmehr von Einschätzungen innerhalb der Gesellschaft ab. Dabei herrscht unter SozialwissenschaftlerInnen keinesfalls Einigkeit darüber, ob Risiken „objektiv“, also real vorhanden oder als Ergebnis einer gesellschaftlichen Konstruktion zu begreifen sind. Die Auseinandersetzung zwischen den Vertretern des „Risiko-Objektivismus“ und des „Risiko-Konstruktivismus“ hält bis heute an (Krohn und Krücken 1993, 9). Beck (1986) versucht, in seinem Ansatz beide Positionen zu integrieren. Für ihn sind die ,neuen Risiken“ sowohl 
gesellschaftlich konstruierte als auch reale Bedrohungen. Andere WissenschaftlerInnen, so beispielsweise Mary Douglas oder Niklas Luhmann, vertreten eine strikt konstruktivistische Perspektive auf Risiken. Insgesamt führte das „Einmischen“ der Sozialwissenschaften in die Risikodebatte zu einer Erweiterung um die soziale(n) Dimension(en) der Risikodefinition und damit zu einer Akzentverschiebung im gesellschaftlichen Selbstverständnis. Ebenso wie in der Öffentlichkeit wird auch innerhalb der sozialwissenschaftlichen Disziplin die Frage, worin der besondere Charakter ,neuer“ - hier verstanden als technik- und wissenschaftsinduzierter - Risiken eigentlich besteht, intensiv diskutiert. Einige unterschiedliche Ansätze sollen hier kurz skizziert werden.

Perrow differenziert zwischen „einfachen Risikohandlungen“ und „komplexen Risikosystemen“ (Perrow 1984, zitiert nach Bonß 1991, 272), wobei letztere sich durch eine ,Vielfalt eng gekoppelter und schneller Prozesse zwischen einzelnen Teilsystemen auszeichnen“ (ebd.) und stets unerwartete Interaktionen auftreten könnten. Charakteristisch für „komplexe Risikosysteme“ ist nach Perrow, dass eine vollständige Risikoüberprüfung aufgrund des hohen Schadenspotenzials zwar notwendig wäre, wegen der systemimmanenten Kontingenzen aber nicht mehr möglich sei.

Für Beck kennzeichnen „moderne“ - in Abgrenzung zu „,vormodernen“ - Risiken deren globale Wirkungen und die soziale Dimension. Im Prinzip könne weltweit jeder Mensch davon betroffen sein. Dies gelte nicht nur für die heutige, sondern auch für künftige Generationen. Weitere Charakteristika „moderner“ Risiken sind für Beck die fehlenden Kompensationsmöglichkeiten im Falle eines Schadens, die Irreversibilität der verursachten Schäden und die Unmöglichkeit, die jeweiligen Verantwortungen einzelnen Personen oder Organisationen zuzuschreiben. Kurz gesagt: Die Wirkungen seien weder örtlich, noch zeitlich oder sozial eingrenzbar. Das mit diesen Eigenschaften einhergehende enorme Schadenspotenzial moderner Risiken lässt nach Ansicht Becks allein ihre Vermeidung zu und so lautet seine Schlussfolgerung: „Risiken meinen eine Zukunft, die es zu verhindern gilt“" (Beck 1986, 44).

Lau differenziert zwischen „traditionellen“ und „neuen“ Risiken. Der wesentliche Unterschied zwischen diesen beiden Risikotypen besteht für ihn darin, dass es sich bei letzteren um zunehmend „diffuse Gefahren handelt, also keine strengen Kausalzusammenhänge nachweisbar sind“ (Lau 1989, 419), woraus sich wiederum das Problem der fehlenden Zurechenbarkeit von Verantwortung ergibt. Unbestimmt blieben auch Art und Umfang der möglichen Schäden, der Zeitraum, in dem sie entstehen und wer davon betroffen sein wird. Im Gegensatz zu „traditionellen Risiken“, die nicht nur individuell zurechenbar und zeitlich begrenzt seien, sondern auch bewusst und freiwillig eingegangen werden - und teilweise sogar Gemeinschaft stiftenden Charakter haben -, sei man von „,neuen“ Risiken unfreiwillig betroffen. Das hieße jedoch nicht, dass sie quasi „naturgegeben“ sind, sondern - so paradox dies zunächst klingen mag - ,durch Entscheidungen und das Handeln von Individuen oder Institutionen verursacht wurden“ (ebd. 423).

Bonß, für den dieser Begriff, anders als beispielsweise bei Beck, nicht per se negativ besetzt ist, beschreibt „Risiko“ als „,spezifischen Typus sozialen Handelns - nämlich ein Handeln unter den Bedingungen der Unsicherheit“" (Bonß 1991, 261). Vom Handelnden würde dieses Risiko bewusst, also freiwillig, in der Erwartung eingegangen, dass sich daraus eine Vervielfältigung der eigenen Handlungsmöglichkeiten ergibt. Bonß unterscheidet jedoch zwischen ,,alten“ und „,neuen“ Risiken beziehungsweise zwischen „Gefahren erster Ordnung“ und „Gefahren zweiter Ordnung“. Letztere kennzeichnet im Kern, dass ,bestimmte Handlungen zeitlich, sozial und/oder sachlich versetzte Nebenfolgen haben, die vorab nicht absehbar sind, das zugrunde gelegte Risikokalkül sprengen und dem Verursacher kaum zugerechnet werden können“" (ebd. 265). Die entstehenden Kosten ließen sich nicht kompensieren und schlügen auf Handelnde und Betroffene gleichermaßen zurück.

Durch Erfahrungen mit den Folgen und Nebenfolgen der Anwendung risikobehafteter Technologien haben sich in der gesellschaftlichen Thematisierung - wie auch in den wissenschaftlichen Risikokonzepten - nicht nur die Wahrnehmung von „Risiken“ und „Gefahren“, sondern auch die 
Ansprüche an den institutionellen Umgang mit diesen gewandelt. Diese Forderung schließt sowohl eine Neugestaltung der Definitionsverhältnisse als auch die Veränderung von Bewertungs- und Entscheidungsstrukturen wie beispielsweise die stärkere Beteiligung der Öffentlichkeit mit ein, da diese aufgrund der enormen Reichweite potenzieller oder faktischer Schäden zunehmend betroffen sei. Lau (1989) kommt zu dem Schluss, dass bisher übliche Bewertungsmaßstäbe und Kompensationsmechanismen sind nicht mehr angemessen seien. Dadurch würde nicht zuletzt die bisher allgemein anerkannte Prämisse, „Risiken als Preis für den Fortschritt erscheinen (zu) lassen“ (ebd. 424) hinfällig. Daraus folge nicht nur ein zunehmender Legitimitätsverlust der wissenschaftlichen Einrichtungen, die bisher die Definitionsmacht für sich beanspruchen, sondern auch ein wachsender Druck auf politische Institutionen, ihre bisherige Praxis im Umgang mit Risiken zu reflektieren und den neuen Ansprüchen anzupassen (Beck 1986).

In der Tat scheint sich mittlerweile auf politischer und wissenschaftlicher Ebene ein Wandel zu vollziehen. Die Strategien zum Umgang mit Risiken beginnen sich - angesichts der sichtbar gewordenen Grenzen der wissenschaftlich-technischen Beherrschbarkeit - zu verändern. So betont der Wissenschaftliche Beirat der Bundesregierung Globale Umweltveränderungen die Bedeutung der Wahrnehmungsmuster, des intuitiven Risikoverständnisses und des jeweiligen Kontextes für die Abwägung und Bewertung von Risiken (WBGU 1999, 41). Berücksichtigt werden solle weiterhin das jeweilige gesellschaftliche Mobilisierungspotenzial, beispielsweise im Falle wahrgenommener Verteilungsungerechtigkeiten. Wiederholt wird das hohe Maß an Ungewissheit, mit dem sich Entscheider im Kontext von systemischen Risiken konfrontiert sehen, betont. Auch die OECD (2003a), die den Begriff ,systemische Risiken“ in die aktuelle Risikoforschung eingebracht hat, hält ein grundlegend anderes Risikoverständnis für erforderlich, um mit „Großkatastrophen“ kompetent umgehen zu können (OECD 2003b, 3). Sie fordert ein „Konzept der integrierten Risikobewertung“, das ein Zusammenwirken verschiedener Disziplinen und die Integration „menschlicher Handlungsweisen - organisatorische(r), kulturelle(r) und soziale(r) Faktoren“ ebenso impliziere wie die Einbindung aller Betroffenen eines Risikofeldes und die Anerkenntnis eines hohen Maßes an Ungewissheit im Umgang mit diesen Risiken (ebd.). Große Bedeutung für einen angemessenen gesellschaftlichen Umgang hat hier auch die Einsicht, dass es eine ,objektive, einheitliche Bewertung" nicht geben könne (ebd., 4).

\subsection{Der Begriff „systemische Risiken“ im Projekt „Risikokonflikte kartiert"}

Der in risikopolitischen Kontexten seit einiger Zeit etablierte Begriff des „systemischen Risikos“ (OECD 2003a, WBGU 1999, Klinke und Renn 2006) wurde eingeführt, um der neuen Qualität der Eingriffs- und Durchdringungstiefe insbesondere durch Unabschließbarkeit und Unüberschaubarkeit Rechnung zu tragen. ${ }^{2}$ Diese neue Qualität zeigt sich im Wesentlichen:

- in zeitlicher Hinsicht aufgrund von Latenzzeiten. So werden die Auswirkungen systemischer Risiken aufgrund der komplexen Vernetzung von Entscheidungen oder Entwicklungen oft erst nach langen Perioden der Latenz spürbar und sind oftmals auf nicht intendierte Nebenfolgen anderweitiger Entscheidungen zurückzuführen;

2 Es mag zunächst contraintuitiv anmuten, gerade Risiken, die sich durch Entgrenzung auszeichnen, als „systemisch“ zu bezeichnen. Sie sind es jedoch insofern, als dass sie potenziell alle Systeme betreffen und aufgrund der komplexen Wirkungszusammenhänge, die die Risiken mit sich bringen, eine nicht kontrollierbare Fülle von Anschlüssen zwischen den Systemen entsteht. 
- in räumlicher Hinsicht, da sie global wirksam sein können und Verursachungs- und Betroffenenkontext auch lokal auseinander fallen;

- in sozialer Hinsicht, da Risiken auf unterschiedliche Teilsysteme durchschlagen, auch künftige Generationen betroffen sind und sie sich in nahezu alle Gesellschaftsbereiche ausdehnen können, die Gesellschaft vordergründig in Entscheider und Betroffene spalten;

- in stofflich-materieller Hinsicht, wenn wir bedenken, dass unsere Gesellschaft als Quasi-Labor fungiert, in dem neue technologische Entwicklungen im Realexperiment erprobt werden. Mehr als einmal hat sich gezeigt, dass sich sowohl Struktur als auch Komplexität (und damit die Eigenschaften) der Risiken selbst wandeln können;

- aber auch in kognitiver Hinsicht, da Ambivalenz und Nichtwissen in der Risikoanalyse eine starke Rolle spielen und unauflösbar erscheinen;

Risikoszenarien erscheinen in Gestalt von weitläufigen, unübersichtlichen Wechselwirkungsketten und zeitigen nicht-intendierte Nebenfolgen, die auf schon getroffene Entscheidungen zurückgeführt werden. Die Zurechnung von kausalen Verantwortlichkeiten wird fast unmöglich.

Aus den beschriebenen Charakteristika von systemischen Risiken ergeben sich Konsequenzen für demokratiepolitisch gestaltete Entscheidungsprozesse und Lösungsstrategien, mit denen die Probleme, die diese aufwerfen, bewältigt werden können. Eine sozial verträglich und demokratiepolitisch orientierte Technikgestaltung muss sich beispielsweise immer der Gefahr einer sozialen Ungleichverteilung der Vor- und Nachteile von Technologien und Technologiefolgen gewahr sein und Strukturen schaffen, die einen gewissen Ausgleich gewährleisten. Um die Interessen aller Betroffenen angemessen berücksichtigen zu können, braucht es partizipative Formen der Aushandlung, wie Technologien gestaltet werden sollen - das reicht bis hin zu Entscheidungen über deren Anwendung bzw. Nichtanwendung - aber auch, wie mit den mit ihnen verbundenen Risiken umgegangen werden soll. Damit kann die Grundlage für politische Entscheidungen verbreitert und können diese von der Gesellschaft stärker mitgetragen werden. 


\section{Das Konzept einer reflexiven Wissenspolitik}

Lange wurde der Wissenschaft ihre Autorität und Fähigkeit, entscheidungsrelevantes Wissen zu erzeugen, nicht streitig gemacht. Dies hat sich jedoch grundlegend geändert. Die Autoritätsansprüche der Wissenschaften und ihr Status als Expertiselieferanten sind schon seit längerem Gegenstand der Diskussion und Kritik (vgl. Wynne 1996, Lévy-Leblond 1999, Felt 2002). So hat Wissenschaft nicht mehr die uneingeschränkte Deutungshoheit darüber, wo die Grenzen zwischen Wissenschaft und Öffentlichkeit, Wissen und Nicht-Wissen, wissenschaftlichem Wissen und anderen Wissensformen, Fakten und Werten verlaufen und welches Wissen entscheidungsrelevant sein soll und welches nicht. Die bisher etablierten Hierarchien zwischen diesen Kategorien werden immer mehr in Frage gestellt und die Grenzziehungen zwischen ihnen neu verhandelt. Auch die Legitimität des Geltungsanspruches der Wissenschaft ist diskutierbar geworden, nicht zuletzt deshalb, weil Wissenschaft ihre angebliche Kontextfreiheit nicht mehr glaubwürdig vermitteln kann. Im Gegenteil, auch Wissenschaft muss die Bedingungen, unter der sie ihr Wissen produziert, immer mehr offen legen und sich eine Befragung durch Öffentlichkeiten gefallen lassen. Die Bedeutung der Wissenschaft als Expertiselieferantin mag zwar nicht generell in Frage gestellt werden - immerhin ist die Nachfrage nach wie vor ungebrochen -, wohl aber sind heutzutage Wissenschaft und ihre Expertisen nicht unumstritten. Gefragt sind also nicht nur „sozial robustes Wissen“ (Nowotny 1999), sondern auch „sozial robuste Verfahren“ der politischen Regulierung (Böschen 2004, 161).

Inzwischen wurde der Blick auch auf andere Wissensformen gerichtet, wie etwa Erfahrungswissen, lokal wirksames und kulturell verankertes Wissen (Irvin und Wynne 1996, Paine 1992) sowie Laien-Expertise (Epstein 1995). Diese Wissensformen werden in ihrem Wert für technologische Entscheidungsprozesse immer mehr anerkannt, zum einen, da sie für mancherlei Problemstellungen Lösungspotenziale bieten, die wissenschaftliche Expertise nicht bereit stellen kann, und, zum anderen, weil sie bisweilen über eine Reflexivität verfügen, die wissenschaftlich erzeugtes Wissen vermissen lässt (Wynne 1993). So hat auch das Vorgehen, nach dem die Politik zur Untermauerung ihrer Entscheidungen auf wissenschaftliche ExpertInnen als „Schiedsinstanz“ (Böschen und Wehling 2004, 11) zurückgreift, seine uneingeschränkte Funktionstüchtigkeit verloren. In diesem Zusammenhang wird die Frage nach einem „,technological“ oder „scientific citizenship“ (Böschen 2004, Irwin 1995, Felt 2003) aufgeworfen. Dabei sollen zivilgesellschaftliche AkteurInnen in die Entscheidungsfindung eingebunden werden, nicht nur, um die Rahmungen der Expertisen auf ihre Sinnhaftigkeit zu überprüfen (Jasanoff 2003) und um technologiepolitische Risikoentscheidungen auf eine breite Basis der gesellschaftlichen Zustimmung zu stellen, sondern auch um damit die Qualitäten anderer Wissensbestände besser zur Geltung zu bringen.

Eine Risikopolitik, die diese Veränderungsprozesse nicht ignorieren will, muss sich daher die Frage nach einer adäquaten Wissenspolitik stellen. Während die regulative Wissenspolitik die „Steuerung“ und „Kontrolle neuen Wissens“ beabsichtigt (Stehr 2003), knüpft die ,reflexive Wissenspolitik“ (Böschen 2005, Wehling 2004a) an das Ziel an, die gesellschaftlichen Aneignungsprozesse von Technologien und Innovationen sozialverträglich und demokratieförderlich zu gestalten und hierfür Verfahren zur Verfügung zu stellen. Reflexive Wissenspolitik ist dabei jenes Feld des Politischen, das zur Institutionenbildung dort beiträgt, wo Form und Legitimität von gesellschaftlichem und wissenschaftlichem Wissen ausgehandelt werden müssen. So werden von verschiedenen Akteuren unterschiedliche Problemwahrnehmungen ins Spiel gebracht, es kursieren unterschiedliche Definitionen dessen, was als legitimes und zu bewältigendes Risiko technischer Innovationen gelten könne und unter welchen Prämissen die Entscheidungsfindung zu diesen Problemen abzulaufen habe (Limoges 1993). Damit diese verschiedensten Perspektiven in die Entscheidungsfindung 
einfließen können und zu ihrem Recht kommen, müssen zunächst die Fragen, wer als legitime(r) ExpertIn gilt - dies können auch LaiInnen sein -, wer das entscheidungsrelevante Wissen generieren kann und darf und wer über diese Legitimität entscheiden darf, Gegenstand öffentlicher Aushandlungsprozesse werden.

Im Rahmen reflexiver Wissenspolitik wird eine Reihe von etablierten Hierarchien explizit gemacht und ansatzweise aufgebrochen. Zum ersten geht es um die Hierarchie zwischen ExpertInnen und LaiInnen. In der Risikopolitik fand sich die Öffentlichkeit lange in der Rolle, sich über das „objektive" Ausmaß eines Risikos von ExpertInnen aufklären zu lassen, mit der Vorstellung, das Vertrauen der Gesellschaft in Technologie werde bei ausreichender Belehrung wieder gefestigt und die Risikobereitschaft erhöht (Wynne 1995, Felt 2002). Öffentlichkeiten sind jedoch nicht mehr nur lernwillige Adressaten von Aufklärung durch wissenschaftliche ExpertInnen, sondern sind in die Produktion von wissenschaftlichem Wissen involviert (Epstein 1995). Damit können und sollten so genannte LaiInnen nicht mehr von vornherein von Entscheidungsprozessen ausgeschlossen werden, so dass das wissenspolitische Feld nicht nur für, sondern auch durch „LaiInnen“ und „Betroffene" geöffnet wird. Reflexive Wissenspolitik gesteht der Öffentlichkeit und deren Akteuren damit eine aktivere, mehr gleichberechtigte Rolle zu als andere Wissenspolitiken.

Die zweite Hierarchie, die im Rahmen einer reflexiven Wissenspolitik neu auszuhandeln ist, ist jene zwischen Wissen und Nichtwissen (Wehling 2004). Nichtwissen gewinnt einen gleich hohen Stellenwert wie Wissen, da gerade in Risikolagen Entscheidungen unter Nichtwissensbedingungen getroffen werden müssen, und Nichtwissen zum prägenden Moment in den Entscheidungsprozessen wird. Mit der Anerkennung der entscheidenden Bedeutung von Nichtwissen geht nicht zuletzt auch die Anerkennung der Notwendigkeit von Lernprozessen im Umgang mit Nichtwissen Hand in Hand.

Zum dritten kann sich eine politische Entscheidung, die sich einer gesellschaftlichen Unterstützung sicher sein will, nicht mehr nur von den so genannten Fakten leiten lassen. Vielmehr müssen auch Wertesysteme und ethische Perspektiven einbezogen werden. Besonders bei den Entscheidungen über risikobehaftete Technologien darf es nicht zur Ausblendung sozialer Wertungen, Erwartungen und Erwünschtheiten kommen, da diese bei Risikobewertungen eine maßgebliche Rolle spielen (vgl. Kapitel 2).

Viertens muss speziell bei wissenschaftlichen Akteuren sichtbar werden, dass Problemdefinitionen und Lösungsstrategien aus verschiedenen disziplinären und damit aus partialen und eben auch beschränkten Perspektiven heraus formuliert werden. Um Expertise unterschiedlicher disziplinärer Provenienz ergänzend und gleichberechtigt behandeln zu können, muss eine reflexive Wissenspolitik diese Partialität und Beschränktheit der wissenschaftlichen Aussagen transparent machen. Denn so lange die unterschiedlichen epistemischen Paradigmen und Kulturen (Knorr-Cetina 1999), die der Wissenserzeugung der jeweiligen Disziplin zugrunde liegen, nicht kritisch mitgedacht werden, wird es immer wieder zu einer nicht gerechtfertigten Höherbewertung naturwissenschaftlicher Expertise gegenüber sozial- und geisteswissenschaftlicher Expertise kommen, die mit dem höheren Faktencharakter ersterer begründet wird. ${ }^{3}$ Diese Bewertungen übersehen, dass naturwissenschaftliches Wissen zwar mit einem höheren - wenn auch umstrittenen - Anspruch an Faktizität antritt als sozial- und geisteswissenschaftliches Wissen, jedoch ebenso einer Partialität unterliegt wie andere Expertisen.

3 Die innerwissenschaftlichen Hierarchien, die - nebenbei erwähnt - auch innerhalb einzelner Disziplinen bestehen, werden im außerwissenschaftlichen Raum aufgegriffen, wie auch umgekehrt außerwissenschaftliche hierarchische Setzungen in die Wissenschaften hineinwirken. 
Als Voraussetzung für einen demokratiepolitischen Entscheidungsprozess über Risikotechnologien bedarf es der Verfahren der Wissenserschließung und -kommunikation über die zur Debatte stehenden Risikostoffe und -technologien. Dabei ist es gerade die Unüberschaubarkeit der verschiedenen Informationen, der erzeugten Erkenntnisse und Aussagen über technische Innovationen und deren Risiken, die die Wissenserschließung notwendig, aber auch gleichermaßen schwierig machen. Die Vielstimmigkeit der sich zu Wort meldenden Akteure, die im Rahmen einer reflexiven Wissenspolitik eingefangen und zugänglich gemacht werden soll, macht die Kommunikation über Innovationen unübersichtlich. Ein Medium der Wissenserschließung zu und Wissenschaftskommunikation über Risikothemen muss daher gleichzeitig auch ein Medium der Komplexitätsreduktion sein. Außerhalb der eigens dafür eingerichteten Instrumente der Risikokommunikation ist ein Großteil der Risikowahrnehmungen und -diskussionen der Dynamik der Massenmedien unterworfen. Mit ihrem „Monopol auf die Fokussierung der Öffentlichkeit“ (Weingart 2003, 120) binden Medien die Aufmerksamkeit des Publikums und bestimmen nicht unwesentlich, welchen Themen öffentliche Aufmerksamkeit gewidmet wird. Problematisiert werden müssen daher auch der $\mathrm{Zu}$ gang zu Wissen und Informationen sowie die Art ihrer Aufbereitung. Nicht nur die Frage, wer das relevante Wissen generiert, ist virulent, sondern auch wer welchen Zugang zu den Informationen, Aussagen und Daten hat, wer von welchen Informationen profitieren kann, wie sie für welchen Zweck verwendet werden und wen der Nichtzugang zu solchen Informationen möglicherweise benachteiligen könnte.

\section{Visualisierung von Risikokonflikten}

\section{I Anforderungen}

In unserem Vorhaben erarbeiten wir eine mögliche Antwort auf die skizzierte Problemlage und schlagen eine internetbasierte Kartierung von Risikokonflikten als ein Werkzeug der reflexiven Wissenspolitik vor. Eine solche Kartierung kann als Argumentationslandkarte auf der Basis von kontroversen Wissenselementen verschiedensten Ursprungs aufgefasst werden. Die Kartierung von Risikokonflikten bildet dabei nicht eine wie auch immer angenommene objektive Wirklichkeit $\mathrm{ab}$, sondern konstruiert einen Wahrnehmungsraum unterschiedlicher Risikoperspektiven.

Um dem Anspruch gerecht zu werden, Einblick in die Situiertheit von Wissenselementen zu geben, die in die Diskurse hineingetragen werden sowie in die Kontextualität der Risikotechnologien, müssen unterschiedliche Wissensformen, disziplinäre Perspektiven und Problemdefinitionen und verschiedenste Akteure in transparenter und sinnfälliger Weise zugänglich gemacht und miteinander verknüpft werden können. Einerseits muss also die Komplexität der Risikofelder deutlich werden, andererseits muss aber auch ein Überblick über die Risikofelder geschaffen werden, um der Unübersichtlichkeit der systemischen Risiken zu begegnen. Um den Anspruch, ein Hilfsmittel der Orientierung zu sein, zu erfüllen, muss eine Kartierung gleichermaßen synoptisch und komplexitätsreduzierend wirken.

Damit eine kartographische Visualisierung auch zur Nutzung anregt, ist der Zugang zu den einzelnen Aussagen und Wissenselementen adressatenfreundlich zu gestalten. Die Internetbasierung gewährleistet zunächst eine allgemeine, zeitlich und örtlich unbegrenzte Zugänglichkeit und Nutzungsmöglichkeit. Darüber hinaus müssen unterschiedlichste Gruppen von NutzerInnen bei ihren verschiedenen Motivationen, Vorkenntnissen, Erwartungshaltungen und Interessen abgeholt wer- 
den, so dass eine unbeabsichtigte Vorabselektion bestimmter Gruppen weitestgehend vermieden wird. Da wir die Kartierung als ein Instrument der Risikokommunikation verstanden wissen wollen, also ein Instrument der Verständigung über die Komplexität, Situiertheit und Dynamik der Konflikte, sollte die Möglichkeit geschaffen werden, dass jeder Nutzer und jede Nutzerin sich als Akteur selbst einbringen kann. Eine Kartierung muss daher auch Elemente zur Interaktion anbieten.

\subsection{Forschungsansatz}

Ein Großteil der schon bestehenden Kartografien von Kontroversen steht der Akteur-NetzwerkTheorie nahe (Latour 2006). Einige dieser Varianten basieren auf automatisierten Verfahren, die etwa Verlinkungsnetzwerke im Internet rekonstruieren, etwa der „Issue-Crawler“ der govcom.orgfoundation ${ }^{4}$. Eine diskursanalytische Erschließung der zu kartierenden Inhalte ist bei diesen Ansätzen jedoch nicht vorgesehen. Dennoch können wir bei der Entwicklung unserer Risikokartierung auf den Erfahrungen der bereits erprobten Kartierungstechniken (siehe z. B. Rogers und Marres 2001, Rogers 2004) aufbauen und zur Weiterentwicklung bestehender Ansätze beitragen.

Unser Zugang zu den Fallstudien ist gleichermaßen durch die Akteur-Netzwerk-Theorie (Latour 1983, Callon 1999) und die Diskursanalyse (Keller et.al. 2001, dies. 2003, Jäger 2001) inspiriert. So begreifen wir die Risikokonflikte gleichermaßen als Diskurse und Netzwerke, an denen nicht nur (menschliche) Akteure beteiligt sind, sondern auch Stoffe, die zur Entwicklung von Kontroversen beitragen. Bei der Materialerschließung, -analyse und auch -interpretation greifen wir auf das methodologische Programm der Diskursanalyse und den theoretischen Ansatz der Netzwerkanalyse zurück. ${ }^{5}$ Im Rahmen der sozialwissenschaftlichen Diskursanalyse werden diskursive Formationen wie etwa Diskurskoalitionen, Narrationen sowie Rahmen- oder Vorläuferdiskurse herauskristallisiert. Beispielsweise wird im Risikodiskurs über nanoskalige Materialien häufig auf Vorläuferdiskurse zurückgegriffen, etwa auf den Asbestkonflikt oder auch auf die Feinstaubdebatte. Auf beide wird von bestimmten Akteuren immer wieder rekurriert, um bestimmte Argumente zu untermauern (vgl. Kapitel 5.1). Darüber hinaus soll auch auf die Historizität der Risikodiskurse und der beteiligten Stoffe aufmerksam gemacht werden (Näheres hierzu siehe Kapitel 6.3).

$\mathrm{Zu}$ den sich am Konflikt beteiligenden Akteuren werden Risikoverständnisse, standortbezogene Beurteilungen, Wissensansprüche und Handlungslogiken rekonstruiert, um damit die Kontextualität und das Eingebettetsein der Akteure zu vermitteln. Das „Risikoverständnis“ spielt darauf an, dass den Argumenten und Aussagen der Akteure unterschiedliche Prämissen darüber zugrunde liegen, was unter welchen Umständen als ein Risiko gelte und wie ihm zu begegnen sei. So argumentieren beispielsweise zahlreiche Akteursgruppen aus einer naturwissenschaftlich-technischen Rationalität heraus, die Risikokonflikte ausschließlich mithilfe naturwissenschaftlich-technischen Wissens zu lösen gedenken. Die unterschiedlichen Bewertungen, die verschiedene Wissensformen damit erfahren, werden durch die Kartierung explizit und hinterfragbar gemacht.

Die zu visualisierenden Informationen und Aussagen werden in den Risikokartierungen in doppelter Hinsicht verfügbar gemacht. Zunächst werden Rohdaten zu den sich einbringenden Akteuren, deren Aktivitäten im Risikodiskurs und Informationen über die beteiligten Stoffe erhoben. An-

4 Siehe_govcom.org-foundation, http://www.govcom.org/scenarios_use.htm (adopted on 17 January 2006).

5 Mit dem diskursanalytischen Ansatz verortet sich unsere Methodik im Paradigma der qualitativen Sozialforschung. Ein zentrales Element des Paradigmas ist die Zurückweisung eines Vollständigkeits- und Objektivitätsanspruches. 
schließend werden Aussagen und Handlungen der beteiligten Akteure und Stoffe diskurs- und netzwerkanalytisch analysiert und interpretiert. Diese Ergebnisse der Diskurs- und Netzwerkanalysen werden gemeinsam mit allen erhobenen Rohdaten für die Einspeisung in die Visualisierungssoftware in einer Datenbankverwaltung abgelegt, wo sie dann softwaregestützt zu einer perspektivischen, geordneten Ansicht zusammengestellt werden (für Beispiele s. Kap. 6.2). Im Ergebnis wird eine Strukturierung des Risikodiskurses als Netzwerk sichtbar, die zu einem weiteren Erkenntnisgewinn über die Risikodiskurse führen kann, welcher ohne softwaregestützte Strukturierung und Visualisierung nicht möglich wäre. Die Einzeldaten werden sukzessive eingegeben und durch die Kartierung derart strukturiert, dass weitere diskursive Muster entdeckt werden können, die bei ausschließlicher Betrachtung der Rohdaten und diskursanalytischen Ergebnisse nicht zu entdecken wären. So werden Muster der gegenseitigen Bezugnahme der Akteure und Sprechakte ablesbar sowie dynamische und aufeinander bezogene diskursive Vernetzungen, wer mit wem koaliert, wer sich gegenseitig das Wort erteilt, wer sich zu welchen Streitfragen äußert, aber auch wer dazu schweigt. Die Ergebnisse der Netzwerk- und Diskursanalyse werden somit über die Kartierung nicht nur abgebildet, sondern die Kartierung selbst erzeugt sozialwissenschaftliches Wissen. Sie ist nicht nur erkenntnisabbildend, sondern auch erkenntnisproduzierend.

In dem hier vorgestellten Kartierungsvorhaben werden exemplarisch zwei ausgewählte systemische Risikofelder - Nanoskalige Materialien und Nahrungsergänzungsmittel - behandelt. Als Querschnittstechnologie macht Nanotechnologie Anleihen in zahlreichen wissenschaftlichen Disziplinen und bringt unterschiedlichste schon etablierte Technologien zusammen. Die neue Qualität der physikalischen und chemischen Eigenschaften von Nanopartikeln ermöglicht einerseits eine Fülle von neuen und neuartigen Anwendungsmöglichkeiten, die von industriellen Werkstoffen über Konsumgüter bis zu medizinischen Anwendungen reichen. Andererseits sind es gerade die speziellen Eigenschaften von Nanopartikeln, deren potentielle unerwünschte Nebenfolgen auf Mensch und Umwelt nicht abschätzbar sind. Die in Betracht zu ziehenden negativen Wirkzusammenhänge in nanotechnologischen Anwendungen sind zu komplex und systemübergreifend, als dass die damit verbundenen Risiken überschaubar oder handhabbar wären.

Nahrungsergänzungsmittel legen ebenfalls die Kategorisierung als systemisches Risiko nahe. Der Trend, dass immer mehr Hersteller Supplemente auf den Markt bringen, ohne dass mögliche negative Effekte geklärt wären, wirft Fragen nach möglichen, gesundheitsrelevanten Wechselwirkungen im Körper auf.

Die zwei Fallstudien repräsentieren unterschiedliche Ausformungen systemischer Risiken. Neben den verschiedenen Inhalten und ihrer verbindenden Eigenschaft, systemische Risiken mit sich zu bringen, unterscheiden sich die Fallstudien in den für sie typischen Dynamiken der Konflikte und darin, wie die „Risikoobjekte“ jeweils konstruiert werden. Prinzipiell können Risikoobjekte sowohl Stoffe als auch Technologien sein, wobei wir unter „Stoffen“ sowohl die Ausgangsstoffe industrieller Produktionsprozesse als auch deren Endprodukte subsumieren. Ferner können aus der risikosoziologischen Perspektive auch potenzielle weitere Charakteristika systemischer Risiken sowie verschiedene Risikoaspekte identifiziert werden - neben ökologischen und gesundheitlichen etwa auch soziale Risiken wie die Veränderung von Machtkonstellationen. 


\section{Die Fallstudien}

\section{I Nanoskalige Materialien}

Die Nanotechnologie gilt in der derzeitigen industriellen wie universitären Forschung und Entwicklung als eine der derzeit wichtigsten technischen Innovationen. Beispiele für heutige und zukünftige Anwendungsfelder der Nanotechnologie sind die Medizin- und Biotechnologie, die Elektronik, die Kosmetikindustrie, der Bereich Neue Materialien, der Automobilbau und der Maschinenbau sowie die Umwelttechnik. Nanoskalige Materialien finden sich auch schon gegenwärtig in zahlreichen Produkten wie Textilien, Reinigungsmittel, Kosmetika und Lebensmitteln wieder, um nur eine Auswahl von Produkten aus der Konsumgüterbranche zu nennen.

Die Versprechungen, die mit der Entwicklung von nanoskaligen Materialien einhergehen, sind zum Teil sehr hoch. Nicht selten ist in diesem Kontext die Rede von epochemachenden Innovationen. Der Grund hierfür liegt in ihren physikalischen und chemischen Eigenschaften, die erst aufgrund der kleinen Größenordnung zum Wirken kommen und nicht der Stoffsorte selbst inhärent sind. Nanoskalige Partikel verhalten sich also aus physikalischen und chemischen Gründen anders als größere Partikel desselben Stoffes. Da sie im Vergleich zur Masse eine größere Oberfläche besitzen als größer dimensionierte Partikel, zeichnen sich viele nanoskalige Materialien durch höhere Härte und extreme Bruchfestigkeit aus, zeigen andere optische und elektronische Eigenschaften und sind chemisch reaktiver.

Wo werden nun die Risiken gesehen? Zum einen werden gesundheitliche Risiken diskutiert, denen Menschen ausgesetzt sind, die mit nanoskaligen Partikeln in Kontakt kommen, sei es über Konsumgüter, im Kontext von medizinischen Behandlungen oder im Rahmen des Arbeitsschutzes in der industriellen Produktion. Hier geht es um die Frage, ob und unter welchen Bedingungen Nanopartikel über die Lunge, den Verdauungstrakt oder die Haut in den Körper und in Folge gar in die Zellen eindringen können und den Organismus schädigen. Das hohe Verhältnis zwischen Teilchenoberfläche und Masse wird häufig als zentrale Ursache für die Toxizität der Nanopartikel angesehen.

Einen zweiten Komplex von Risikofragen bilden die mit der Produktion, Verwendung und Entsorgung von Nanopartikeln verbundenen Umweltrisiken. Hier ist derzeit noch völlig ungeklärt, ob und welche, möglicherweise irreversiblen Schäden in die Umwelt ausgesetzte Nanopartikel bewirken würden. Bei der Argumentation um diese Risiken wird häufig an die Debatten über Feinstäube angeknüpft, die seit wenigen Jahren epidemiologisch und toxikologisch studiert werden und in der Öffentlichkeit starke Aufmerksamkeit erfahren. Weiterhin wird mit dem Rückblick auf die Schädlichkeit von Asbest, die zu lange verkannt wurde, auf potenzielle Risiken ähnlichen Ausmaßes bei Nanopartikeln verwiesen (Swiss Re 2004). Dabei überrascht nicht, dass gerade die Versicherer bei Nanopartikeln diese Parallele ziehen, zumal der Asbestfall für die Versicherungsbranche einen der kostspieligsten Versicherungsfälle der Geschichte darstellt.

Als Charakteristikum der Risikodiskurse über nanoskalige Partikel kann die zentrale Rolle des (Noch-)Nichtwissens gesehen werden, dass also hauptsächlich über Risiken verhandelt wird, die (noch) nicht genau benannt werden können. Kaum jemand bestreitet, dass bei der Herstellung und Verwendung nanoskaliger Materialien Umwelt- und gesundheitliche Risiken nicht ausgeschlossen werden können. Konfliktpotenzial in diesen Diskursen birgt aber die Frage, wie mit dem Nichtwissen über die möglichen Risiken von Nanopartikeln adäquat umgegangen werden soll. Offen ist auch, welche Vorsorge angesichts potenziell eintretender Risikofälle zu treffen sei. Aus diesen zum Teil konfligierenden Ansichten formiert sich ein „Diskurs über den Diskurs“, in dem darüber verhandelt wird, wie die Gesellschaft über die Risiken der Nanotechnologien erfolgreich debattieren könnte. 
Relativ früh in der Etablierung der Nanotechnologien zeichnete sich ein Bewusstsein für die Notwendigkeit öffentlicher Debatten über Nanotechnologien ab, die meist als so genannte ,dialogische Maßnahmen“ eingefordert wurden und werden. Eng damit verwoben ist die Diskussion um die Wünschbarkeit solcher Entwicklungen. Nicht nur Non-Governmental-Organisations, auch Akteure aus dem politischen, dem wirtschaftlichen sowie dem wissenschaftlichen Sektor betonen die Wichtigkeit einer öffentlichen Diskussion über Nanotechnologien. Einigen scheint hier die Einbeziehung von Stakeholdern in die Entscheidungsfindung ausreichend, andere fordern eine Beteiligung unterschiedlicher „Öffentlichkeiten“. Wieder andere - nicht nur Vertreter aus dem sozialwissenschaftlichem Bereich - plädieren mit Nachdruck dafür, dass man aus den Erfahrungen mit den Debatten zur Grünen Gentechnik lernen solle (Parr 2005, Grove-White et.al. 2004). Die erhöhte Sensibilität für die Notwendigkeit öffentlicher Debatten um Nanotechnologien scheinen die Erfahrungen mit den Diskursen um die Grüne Gentechnik mitbewirkt zu haben.

In Anlehnung an den Digital-Divide wird auch die Gefahr eines so genannten „Nano-Divides“ in die Diskussion gebracht. Diese Formulierung bringt zum Ausdruck, dass es in erster Linie die hoch-industrialisierten Nationen, und auch dort nur die materiell begünstigten sozialen Gruppen sind, die sowohl vom Boom in Forschung und Entwicklung wirtschaftlich profitieren können als auch in den Genuss neuer Möglichkeiten der Nanotechnologien, insbesondere im medizinischen und Lifestyle-Bereich kommen. Die Mahner eines Nano-Divides leiten daraus die Forderung nach Verteilungsgerechtigkeit ab.

\subsection{Nahrungsergänzungsmittel}

Der Markt für Nahrungsergänzungsmittel (NEM) boomt. Der weltweite Umsatz mit Nahrungsergänzungsmitteln wird derzeit auf mehr als 1 Mrd. Euro geschätzt (aid 2005, 12). Verglichen mit anderen Risikokonflikten (bspw. Grüne Gentechnik oder Elektrosmog) ist der Konflikt um Nahrungsergänzungsmittel relativ jung. Das manifestiert sich unter anderem in den vergleichsweise neuen und teilweise noch in Entstehung befindlichen gesetzlichen Regelungen. Weiterhin kennzeichnet diesen Konflikt, dass er aktuell nicht in der breiten Öffentlichkeit geführt wird, sondern überwiegend zwischen Politik, Wissenschaft und Unternehmen, teilweise auch Verbraucherschutzorganisationen stattfindet. Das rasche Marktwachstum für Nahrungsergänzungsmittel der letzten Jahre - ein wesentlicher Auslöser für deren kritische Thematisierung innerhalb oben genannter Akteursgruppen - zeigt, dass die Mehrheit der VerbraucherInnen Nahrungsergänzungsmittel bisher offensichtlich nicht als Risiko wahrnimmt. Im Gegenteil: Die auch als „Gesundheitslebensmittel" bezeichneten Produkte werden oft aus Angst vor Mangel- und Fehlernährung konsumiert - also um ein gesundheitliches Risiko zu mindern (Hahn et.al. 2004).

Charakteristisch für den Konflikt um Nahrungsergänzungsmittel ist die große Bedeutung der Auseinandersetzungen um den geeigneten rechtlichen Rahmen. Ein wesentlicher Grund hierfür sind die zahlreichen Unsicherheiten, die sich aus der aktuell ,fluiden“ und deswegen oft unklaren Rechtslage und den lückenhaften Regelungen ergeben. Allein schon die Frage, was unter Nahrungsergänzungsmitteln eigentlich zu verstehen sei, ist sowohl auf rechtlicher als auch auf wissenschaftlicher Ebene Gegenstand der Auseinandersetzung. Die fehlende Klarheit des Begriffs zeigt sich unter anderem in der relativ offenen Definition in der aktuell in Deutschland gültigen Nahrungsergänzungsmittelverordnung (NemV). Nahrungsergänzungsmittel (NEM) sind hier definiert als „Lebensmittel, die dazu da sind, die allgemeine Ernährung zu ergänzen“, stellen ein „Konzentrat von Nährstoffen dar und werden in dosierter Form (beispielsweise als Kapseln, Pillen, Tabletten oder in Pulverform) angeboten“" (BMVEL 2004, 1011). Außerdem umfasst die Verordnung bisher nur einen kleinen Ausschnitt aller Stoffe, die in Nahrungsergänzungsmitteln enthalten sein können. Ungeregelt ist bis- 
her auch die jeweilige Konzentration des Nährstoffs, der in einem Produkt enthalten sein darf (Verbraucherzentrale 2005, 21). Zur Debatte steht eine ganze Reihe von weiteren Aspekten, so beispielsweise die Frage, mit welchen Aussagen zu den Wirkungen NEM beworben werden dürfen. Des weiteren wird die schwere Unterscheidbarkeit zwischen Nahrungsergänzungsmitteln und Arzneimitteln kritisiert, die dazu führen könnte, dass VerbraucherInnen Nahrungsergänzungsmittel quasi als Medikament einsetzen, obwohl diese Produkte als Lebensmittel definiert sind und - anders als Pharmaka - weder zugelassen noch geprüft werden müssen, bevor sie auf den Markt kommen.

Kontrovers diskutiert wird auch, ob und zu welchem Zweck Nahrungssupplemente eingenommen werden sollten, wobei eindeutige pro- oder contra- Haltungen eher die Ausnahme sind. Eine häufig vertretene Position in den Reihen der Skeptiker lautet beispielsweise, NEM seien prinzipiell überflüssig, aber für bestimmte Risikogruppen oder in bestimmten Lebenssituationen könne ihre Einnahme durchaus sinnvoll sein. Uneinigkeit herrscht auch darüber, welche NEM zum Einsatz kommen sollten. Dabei sind die jeweiligen Bewertungskriterien und -maßstäbe und die daraus resultierenden Kategorisierungen akteursspezifisch sehr unterschiedlich. In Abhängigkeit der jeweiligen Vorstellungen von ,richtiger Ernährung“, dem als relevant erachteten Wissen zur Bewertung von Nahrungsmitteln und den spezifischen Interessen der jeweiligen Akteursgruppen erfolgt die Bewertung danach, ob sie natürlich“ oder „künstlich“ hergestellt wurden, ob es sich um ,echte“ oder „,unechte“ NEM handelt oder ob sie, ,wissenschaftlich geprüft“ oder , ungeprüft“ sind.

Eine wesentliche Rolle spielt auch die Frage der gesundheitlichen Risiken. Die meisten in Nahrungsergänzungsmitteln eingesetzten Stoffe als solche sind nicht neu - sondern sogar notwendiger Bestandteil unserer Ernährung. Der wesentliche Unterschied besteht in der Art und Weise der Herstellung und der Form, in der man sie zu sich nimmt. Was von den einen als große „Stärke“ der Supplemente beschrieben wird - dem Körper gezielt die von ihm aktuell benötigte Menge an Nährstoffen zuzuführen - stellt für die anderen genau das „Riskante“ dar. Die aus dem Zusammenhang gelöste Aufnahme von Einzelnährstoffen stelle demnach etwas völlig anderes dar als ihr Konsum über herkömmliche Lebensmittel. Risiken, die sich daraus ergeben, werden unter anderem in möglichen Fehl- oder Überdosierungen, Wechselwirkungen mit anderen Stoffen und möglichen schädlichen, bisher unbekannten Wirkungen im Körper gesehen. Auch die Qualität der Produkte selbst wird problematisiert, wie etwa die schwankende Zusammensetzung und Konzentration der Inhaltsstoffe sowie mögliche toxische Belastungen. Risiken könnten sich auch aus „überzogenen“ Hoffnungen auf die Wirkung der Produkte ergeben, die wiederum dazu führen könnten, dass eigentlich notwendige medizinische Maßnahmen unterlassen würden aus der Hoffnung, mit Hilfe einer ,wohldosierten“ Zufuhr von Nährstoffen die Folgen einer „ungesunden“ Lebensweise (unter anderem Zigarettenkonsum) ausgleichen zu können.

Ökonomische Risiken, die sich aus den Veränderungen des Marktes der letzten Jahre ergeben, werden ebenfalls thematisiert. Dazu gehört neben der zunehmenden Vielfalt der Einkaufsstätten die wachsende Diversität der Anbieter. So sind beispielsweise Unternehmen aus der Pharmabranche mittlerweile sehr aktiv in dieser Branche. Ein quasi explodierendes und ständig wechselndes Angebot macht es für VerbraucherInnen fast unmöglich, sich eine Marktübersicht zu verschaffen.

Insgesamt wird immer wieder auf die schlechte Datenlage und fehlende wissenschaftliche Untersuchungen sowohl potenzieller positiver wie auch negativer Wirkungen hingewiesen, die eine Bewertung von Stoffen und Produkten zum gegenwärtigen Zeitpunkt nicht zulassen oder nur vorläufige Bewertungen erlauben (Diedrich 2002, Hahn et.al. 2006).

Der systemische Charakter des Risikos zeigt sich zunächst in der Vielzahl der ungeklärten Fragen - sei es hinsichtlich möglicher gesundheitlicher Auswirkungen, hinsichtlich des rechtlichen Rahmens oder hinsichtlich der Marktsituation. Verstärkt wird er durch die Wechselwirkungen zwischen diesen Bereichen: So können aufgrund unzureichender gesetzlicher Regulierungen Produkte auf den Markt kommen, deren Wirkungen nicht untersucht oder die für VerbraucherInnen nicht transparent sind und daher gesundheitliche Risiken darstellen. 


\section{Bisherige Überlegungen zur Visualisierung}

\section{I Die Kartierungslogik}

Im Sinne einer reflexiven Wissenspolitik muss es, wie oben dargestellt, also darum gehen, mit Hilfe einer kartografischen Darstellung möglichst vielen Beteiligten den Zugang zu relevantem Wissen zu verschaffen sowie gleichzeitig einen Eindruck von der Komplexität und Dynamik des Konfliktes zu vermitteln - und dies auf eine Weise, die nicht verwirrend und damit abschreckend wirkt, sondern zur Teilnahme am Diskurs motiviert. Anhand erster Entwürfe soll hier deutlich gemacht werden, wie diesen Ansprüchen in der praktischen Umsetzung Rechnung getragen wird.

Der Einstieg in die Karte erfolgt über die Fallbeispiele. Die Fallstudien werden entsprechend der exemplarischen Vorgehensweise zwar unabhängig voneinander, aber nach den gleichen Visualisierungsprinzipien dargestellt. Dabei wurden die Zugänge zu den Fallstudien direkt in die Kartierungslogik übernommen. Denn dies entspricht sowohl der methodischen Aufbereitung als auch den Interessen vieler NutzerInnen. Dazu gehören in etwa die Fragen, ob ein Stoff risikobehaftet ist, wer zu den Befürwortern und Gegnern in einer bestimmten Streitfrage gehört und mit welchen Formulierungen sich jemand am Diskurs beteiligt. Daraus ergeben sich zunächst drei Perspektiven zur Abbildung der Diskurse:

- Zum einen auf die Akteure, die sich in die Diskurse einbringen. Dazu kartieren wir Sprecher und deren Aussagen und Aktivitäten;

- Zum zweiten, auf Stoffe beziehungsweise Produkte und Technologien, die beteiligt und Gegenstand der Diskurse sind;

- Zum Dritten, auf die Streitfragen, um die es geht. Damit sind Thematisierungen von Risiken gemeint, über die Uneinigkeit herrscht, also „gestritten“ wird.

Als Grundstruktur der Seiten wurde - wie Abbildung 1 (auf der nächsten Seite) zeigt - eine Einteilung in drei Bereiche gewählt. In der Mitte befindet sich der eigentliche Visualisierungsbereich bzw. die graphische Umsetzung des Risikokonfliktes. Die einzelnen Elemente werden durch Icons in Form von unterschiedlich gestalteten Fähnchen symbolisiert. In der „Info-Box“ daneben finden sich Erläuterungen (beispielsweise warum und mit Hilfe welcher Methoden diese Ansicht erstellt wurde) und Hintergrundinformationen. Am oberen Rand ist ein Navigator platziert, der der Orientierung der NutzerInnen dienen soll, also Auskunft darüber gibt, wo sie sich in der Karte gerade befinden und welche Möglichkeiten sie haben, die jeweilige Ansicht zu verändern. Von hier aus haben NutzerInnen vielfältige Möglichkeiten, sich ihren eigenen Weg durch die Karte zu suchen und sich den Konflikt seinen persönlichen Interessen entsprechend zu erschließen. Er/sie kann auf diese Weise Ansichten selbst konstruieren und je nach Bedarf die Perspektive wechseln. So kann man beispielsweise auf ein Icon klicken, um sich in der Info-Box Informationen zu dem jeweiligen Element zeigen zu lassen. Der Einstieg über die verschiedenen Objekte (Akteure, Stoffe, Streitfragen) stellt einen weiteren möglichen Zugang dar. Dabei sind diese „Objekte“ nicht unabhängig voneinander kartiert, sondern im Hintergrund miteinander vernetzt.

Im Sinne einer motivierenden Darstellung ist es wichtig, als NutzerIn Komplexität „dosieren“ zu können, beispielsweise durch den Wechsel zwischen „Komplettansichten“ und „einfachen Ansichten", beziehungsweise zwischen Gesamt- und Detailansichten wie durch das gezielte Heranzoomen einzelner Elemente (beispielsweise eines Akteurs oder eines Stoffes). Die Komplexität der Ansicht wächst auch mit dem sukzessiven Sichtbarwerden von Vernetztheit, die mit Hilfe des „Networkers" hergestellt werden kann. Dieses zusätzliche Instrument ermöglicht es, die Aufeinanderbezogenheit einzelner Elemente aufzuzeigen. Auf diese Weise kann man beispielsweise der Frage 
nachgehen, welcher Akteur sich zu welchen Streitfragen mit welchen Argumenten äußert. Darüber hinaus ist es möglich, zu verfolgen, in welchem Kontext die Äußerung gemacht wurde. Damit wird gleichzeitig sichtbar, welche Aktivitäten (beispielsweise Konferenzen, Publikationen von Broschüren, Bürgerinitiativen) initiiert werden, um der eigenen Position Gehör zu verschaffen. Auch die $\mathrm{Zu}-$ sammenhänge zwischen gleichen Objekten - wer mit wem koaliert, welcher Akteur von einem anderen finanziell unterstützt wird oder wie einzelne Aussagen miteinander verbunden sind (ob sie sich gegenseitig unterstützen oder widersprechen) - kann mit Hilfe des „Networkers“ angezeigt werden.

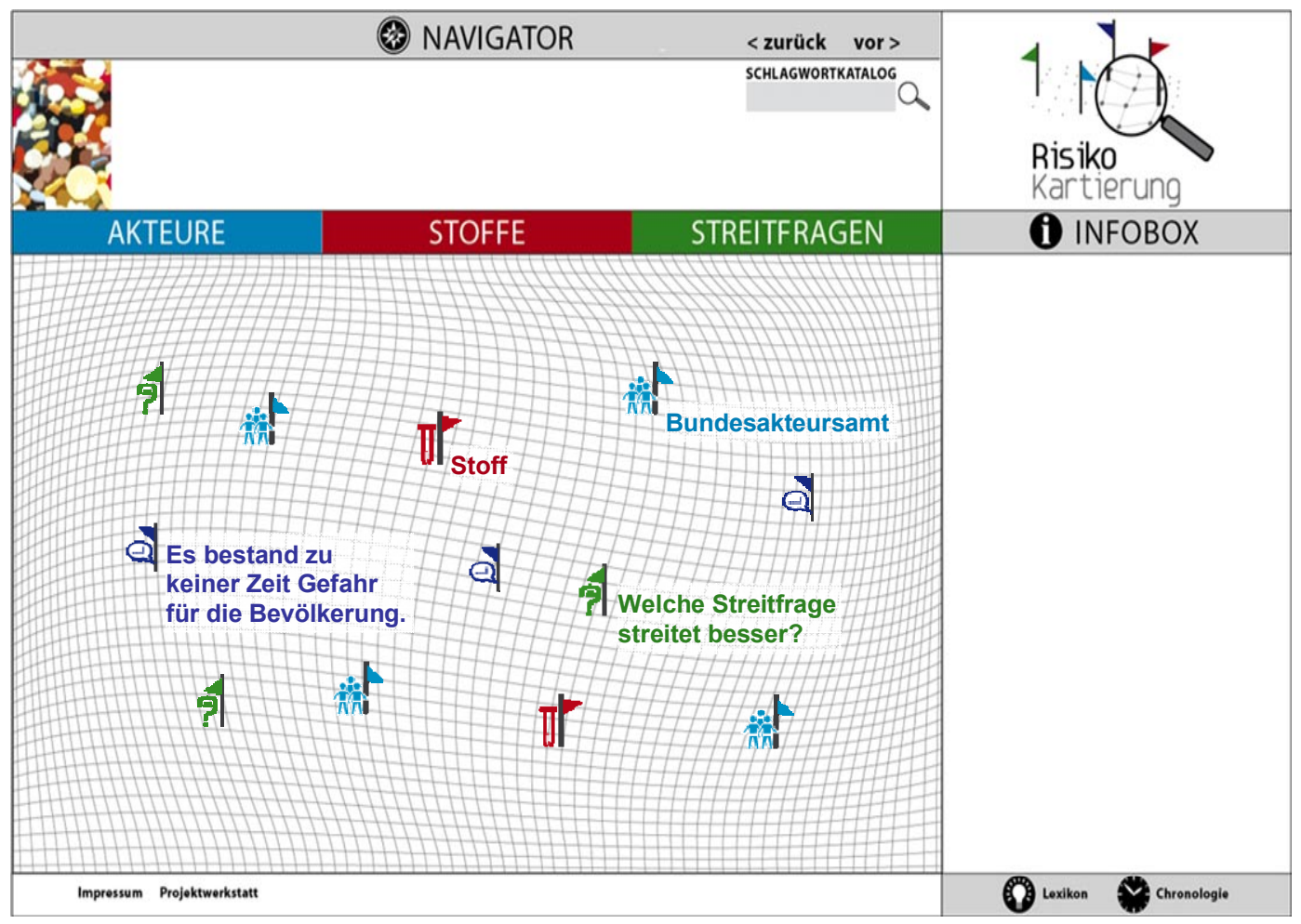

Abbildung 1: Visualisierungsentwurf „Übersicht“

Eine weitere Möglichkeit, den unterschiedlichen Bedürfnissen der NutzerInnen (so beispielsweise „Schnell-LeserInnen“ und „Quasi-ExpertInnen“) Rechnung zu tragen, stellt das Anbieten verschiedener Informationsebenen dar, die sich in Umfang und Detailliertheit der angebotenen Information unterscheiden, aber wiederum direkt miteinander verknüpft sind. In der Karte wird dies etwa durch die Verbindung des Visualisierungsbereichs mit der Info-Box, von der wiederum auf ausführlichere Texte oder Originaldokumente durchgegriffen werden kann, umgesetzt.

Ein weiteres Instrument in der Kartierung ist die so genannte „Werkstattseite“. Diese enthält reflexive Elemente, das heißt hier wird Transparenz über unsere Forschungsinteressen, unsere methodische Vorgehensweise und Entscheidungsverfahren, etwa in Bezug auf inhaltliche Beschränkungen, Selektionen und Interpretationen, hergestellt. Interaktive Elemente sollen es den NutzerInnen ermöglichen, das Gesehene zu kommentieren und Anregungen für die Weiterentwicklung zu platzieren. 


\subsection{Strukturelemente der Kartierung am Beispiel Nahrungsergänzungsmittel}

Ein wichtiges Gestaltungselement im Rahmen der Kartierung ist die Strukturierung der Elemente, d. h. die Gruppierung anhand bestimmter Kriterien - hier auch „Ordnungen“ genannt. Solche Ordnungen können in mehrfacher Hinsicht nützlich sein. Zunächst dienen sie der Orientierung, weil sie es den NutzerInnen erleichtern, einen Überblick über ein komplexes Feld zu bekommen. Darüber hinaus können solche Strukturierungen den „Einstieg“ in das Risikofeld erleichtern, weil sie dem persönlichen Zugang des/der jeweiligen NutzerIn entsprechen (z. B. Anordnung der Akteure nach Funktionsbereichen) oder es ermöglichen, einen spezifischen Ausschnitt aus der Debatte relativ schnell auswählen und betrachten zu können (zum Beispiel in welchen Streitfragen über gesundheitliche Risiken gesprochen wird). Verstanden als didaktisches Element bieten diese Ordnungen die Möglichkeit, bestimmte Kontexte abzubilden oder bestimmte Perspektiven, die im Hinblick auf die Darstellung des Risikokonfliktes von besonderem Gewicht zu sein scheinen, zu entwickeln (in etwa diskursive Rahmungen). Vorstrukturierte Ansichten stellen also eine Möglichkeit dar, sowohl im Sinne einer nutzungsgerechten Gestaltung auf die (vermuteten) Bedürfnisse der NutzerInnen einzugehen als auch die Anliegen des Projektes besser zu vermitteln. Da Ordnungen eine starke Interpretation durch die KartiererInnen darstellen, müssen sowohl Methode als auch inhaltliche Begründung an geeigneter Stelle in der Karte angegeben werden (um für die NutzerInnen nachvollziehbar zu sein).

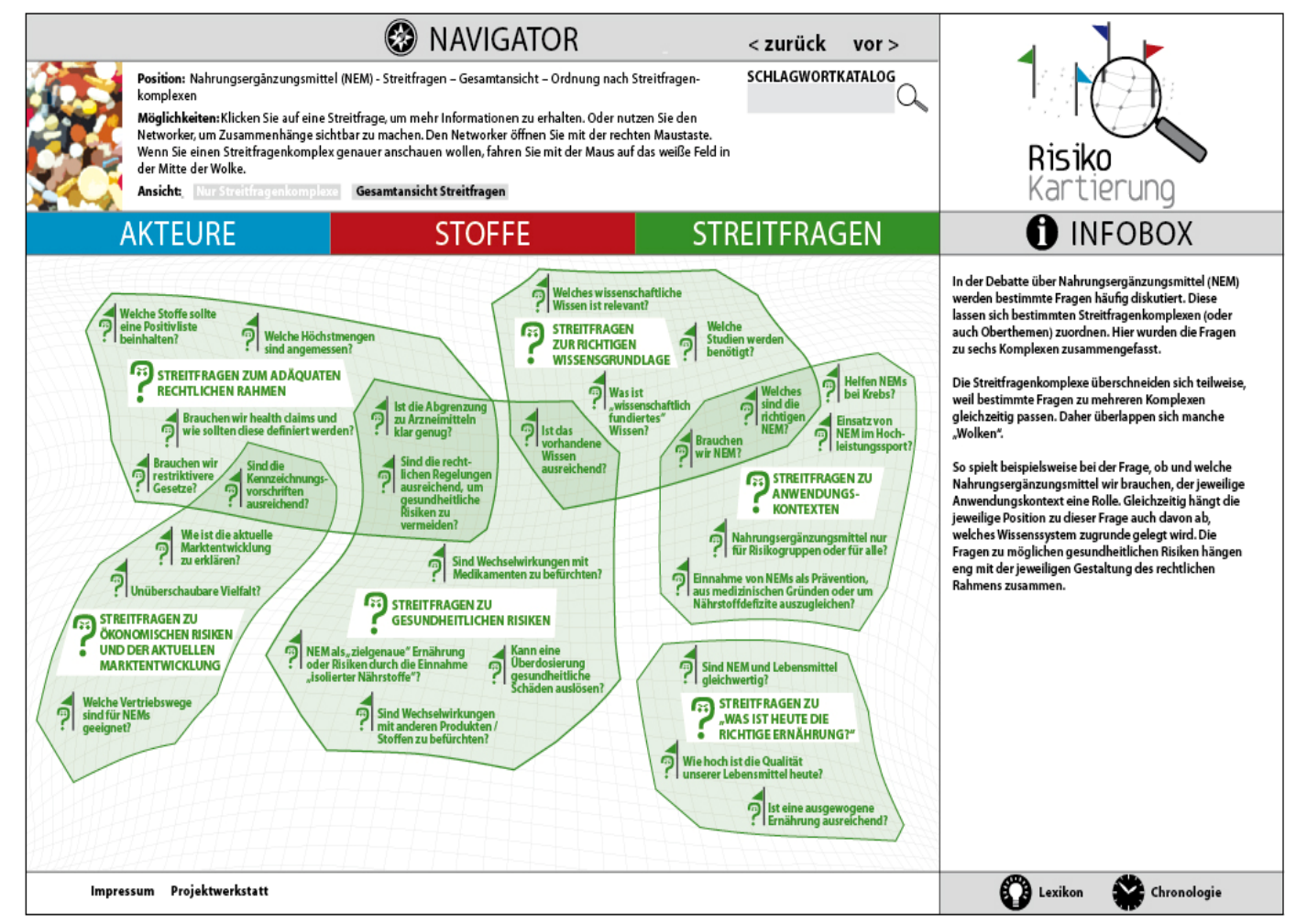

Abbildung 2: Visualisierungsentwurf: „Ordnung nach Streitfragenkomplexen“ 
Exemplarisch wird in Abbildung 2 eine Ordnung der Streitfragen dargestellt. In dieser Ansicht wurden die im Rahmen der Diskursanalyse identifizierten Streitfragen in „Komplexen“ (nach thematischer Nähe) angeordnet. ${ }^{6}$ Dies soll es den NutzerInnen ermöglichen, einen etwas übersichtlicheren Gesamteindruck darüber zu bekommen, über was hier gesprochen bzw. gestritten wird und leichter solche Fragen oder Fragenkomplexe zu finden, die sie besonders interessieren. Die Überlappungen zwischen den einzelnen Wolken symbolisieren die Vernetzung zwischen einzelnen Komplexen im Diskurs. Denn viele Streitfragen sind in verschiedenen thematischen Kontexten relevant. So ist beispielsweise die Frage, welche Nahrungsergänzungsmittel wir benötigen, sowohl von der zu Grunde gelegten Wissenslogik (,Welches Wissen ist in diesem Kontext relevant?“) als auch vom jeweiligen Anwendungskontext abhängig. Da die meisten Überschneidungen zwischen den Streitfragen zu Risiken und anderen Komplexen existieren, wurde dieser Komplex hier ins Zentrum gerückt. Dies verdeutlicht, dass die Frage möglicher Risiken bei fast allen Teilaspekten der Debatte eine Rolle spielt und bestätigt damit indirekt die Intention des Projektes, das den Fokus auf die Darstellung der jeweiligen Risikoaspekte richtet. Um bei den NutzerInnen das Gefühl der Überforderung zu vermeiden, werden an dieser Stelle nicht alle Streitfragen angezeigt, sondern zu jedem Komplex nur eine gewisse Auswahl. Jedoch haben NutzerInnen die Möglichkeit, bei Interesse alle Streitfragen in einer ungeordneten Ansicht anzuschauen. In der Info-Box findet man einen erläuternden Text zu der hier abgebildeten „Streitfragenlandschaft“. Unter anderem kann man hier erfahren, warum diese Anordnung gewählt wurde oder was an dieser Anordnung für die jeweilige Fallstudie charakteristisch ist - so in der Fallstudie Nahrungsergänzungsmittel das relativ große Gewicht der rechtlichen Fragen und der Fragen zur Marktentwicklung. Gegebenenfalls können hier auch Erläuterungen zu den einzelnen Komplexen stehen. Weitere Möglichkeiten für solche strukturierten Darstellungen sind die Ordnung der Akteure nach ihren Funktionsbereichen (Wirtschaft, Politik, Öffentlichkeit und Wissenschaft), nach ihrer Haltung zum Risikokonflikt oder die Zuordnung von Aussagen zu relevanten diskursiven Rahmungen. Analog zu den Streitfragenkomplexen bietet sich auch hier die Gruppierung in Wolkenform an (ähnlich wie in Abbildung 2).

\subsection{Die Chronologie der Diskurse am Beispiel der Nanopartikel-Debatte}

Neben einer strukturierten Darstellung aller Akteure, Stoffe und Streitfragen zum ausgewählten Risikodiskurs soll auch die Historizität von Risikodebatten vermittelt werden, denn die Risikodiskurse und die ihr zugrunde liegenden Technologien sind nicht ahistorisch, sondern in historische Entwicklungen eingebettet. Die zeitliche Dimension der Risikodiskurse kann im Rahmen der eigentlichen Risiko-Kartierung nach Akteuren, Stoffen und Streitfragen gemäß der oben beschriebenen Struktur jedoch nicht transportiert werden, da das chronologische Aufeinanderfolgen von Aussagen und Wortmeldungen zu den Risikodebatten in der eigentlichen Risikokartierung nicht zum Ausdruck kommt. Unabhängig vom Kartierungsfenster wird daher eine Chronologie angeboten, die zur jeweiligen Fallstudie jederzeit aufgerufen werden kann. Bei der Visualisierung der Chronologie besteht ein entscheidender Unterschied zur eigentlichen Risiko-Kartierung darin, dass die Seiten innerhalb der Kartierung durch die Anfragen der NutzerInnen über die Software erst aufgebaut werden (z. B. über die Anfrage, sich alle Akteure anzeigen zu lassen, die sich zu einer bestimmten Streitfrage geäußert haben). Beim Aufrufen der Chronologie werden dagegen die Inhalte

6 Die Texte in der Abbildung sind das Ergebnis erster Recherchen und haben daher vorläufigen Charakter. Sie wurden hier trotzdem verwendet, um das Konzept der Visualisierung zu veranschaulichen. 
nicht über Software errechnet, sondern bestehen aus vorab eingegebenen Texten, die in 2 bis 3 Sätzen die einzelnen geschichtlichen Stationen erläutern und auf einschlägigen Veröffentlichungen basieren. Die graphische Anordnung der jeweiligen Chronologie-Einträge wird von der Fallstudien-Bearbeiterin vorgegeben.

Mit der Chronologie sollen NutzerInnen sich einen Eindruck über die Geschichte der jeweiligen Risikotechnologie verschaffen können. Für die Fallstudie über nanoskalige Partikel beispielsweise soll sie einen Überblick bieten, wann welche Entdeckungen gemacht und Entwicklungen von statten gegangen sind, die von vielen Akteuren als entscheidend angesehen werden und die gemeinhin als Baustein der „Geschichte der Nanotechnologie“ interpretiert werden. Die Diskursgeschichte konstituiert sich dabei nicht unwesentlich über den Begriff „Nano“. Daher ist bei der Formulierung „Geschichte der Nanotechnologie“ nicht nur an eine Aufzählung der Stationen zu denken, an denen bestimmte Verfahren zur Herstellung und Manipulation von nanoskaligen Partikeln erstmals erprobt und umgesetzt wurden, sondern es werden darunter sowohl die sukzessive Etablierung des Begriffes „Nanotechnologie“ subsumiert als auch die zentralen Stationen der Risikodiskurse um Nanotechnologien.

Aber die Vorstellung einer „Geschichte der Nanotechnologie“ muss gleichzeitig problematisiert werden, denn von einer „Geschichte der Nanotechnologie“ kann man genau genommen erst ab dem Zeitpunkt der Etablierung des Begriffes sprechen. Insbesondere einige Ereignisse im vorigen Jahrhundert wurden ex post zu den so genannten „Anfängen“ der Nanotechnologie stilisiert und als Baustein in der Geschichte der Nanotechnologie konstruiert. So wird z. B. Richard Feynman aufgrund seiner Rede von 1959, in der er die gezielte Manipulation von Atomen und Molekülen für möglich erklärt, heutzutage als Vordenker und Visionär der Nanotechnologie bezeichnet. Andererseits hat man schon in früheren Jahrhunderten Partikel mit nanoskaligem Ausmaß hergestellt und sich systematisch zu Nutze gemacht, lange bevor Nanotechnologie als ,Technologie des 21. Jahrhunderts“ ausgerufen wurde. Diese Verfahren werden jedoch gemeinhin auch heutzutage nicht der Geschichte der Nanotechnologie zugeordnet. Weiterhin werden seit den 40er Jahren nanoskalige Materialien in industriellem Maßstab hergestellt und verbreitet. Der Risikodiskurs zu Nanopartikeln, der eben auch diese Materialien betrifft, hat sich jedoch erst seit Deklarierung der „Nanotechnologien“" entwickelt.

Als weiteres Ziel der Chronologie soll die Triggerfunktion von diskursiven Beiträgen sichtbar werden. Aussagen und Aktivitäten von Akteuren lösen bei anderen Akteuren wiederum neue Handlungen und Meinungsäußerungen aus. Beispielsweise hat die Gründung der Förderungsinitiative der Nanotechnologie NNI in den USA unter anderem mit dazu beigetragen, dass die EU mit dem Argument, nicht den internationalen Anschluss verlieren zu dürfen, mit Strategiepapieren zur Nanotechnologie geantwortet hat.

Um den Verlauf auf einer Zeitachse nutzergerecht sichtbar machen zu können, wird an dieser Stelle auf eine netzwerkartige Visualisierung verzichtet. Das Visualisierungskonzept der Chronologie beruht daher auf separaten Zeitleisten, die beide auf einem Bildschirm erscheinen (s. Abbildung 3 auf der nächsten Seite). Die erste Zeitleiste, die so genannte „Teilchen“-Leiste, spiegelt das Stoffliche wider. Auf ihr werden wichtige Entdeckungen und Verfahren zur Herstellung und Verarbeitung von nanoskaligen Teilchen aufgezeigt. Unter anderem ist hier nachzuverfolgen, dass mit nanoskaligen Partikeln nicht erst seit einigen Jahrzehnten gearbeitet wird. Auf der zweiten Leiste geht es um die Geschichte der Diskurse um nanoskalige Teilchen. Sie zeichnet die historische Entwicklung der Diskurse über „Nanotechnologie“ nach, beginnend mit der erwähnten Rede Feynmans von 1959, gefolgt unter anderem von der Einführung des Begriffes 1974 und seine Etablierung, die sich erst in den 80er Jahren vollzog. Ab dieser Phase Mitte der 80er Jahre entsteht, wie auf der Diskursleiste abzulesen, erst im wörtlich expliziten Sinne die „Geschichte der Nanotechnologie“. Die einzelnen Stationen zum Umgang mit nanoskaligen Materialien konvergieren in der Zeitphase, in der sich die Diskurse zur Nanotechnologie allmählich herauskristallisieren. Die Ein- 
träge der Teilchenleiste vor den 80er Jahren eint daher lediglich, dass sie sich mit Materialien beschäftigen, die nanoskaliges Ausmaß haben. Sie sind jedoch nicht als lineare Abfolge zu interpretieren. Daher sind die Stationen vor Mitte der 80er, die erst rückwirkend als „Vorläufer“ der Nanotechnologie tituliert werden, zwar chronologisch angeordnet, bedingen sich aber nicht im Sinne einer kausal aufeinander aufbauenden „Entwicklung der Nanotechnologie“. Da Stoffe und Akteure, Teilchen- und Diskursgeschichte, nicht in einer einzigen Zeitleiste in linearer Abfolge aufgelistet werden, können so anhand von Querverbindungen, die sich zwischen beiden Leisten entdecken lassen, zwar keine Netzwerke, jedoch Wirkungszusammenhänge zwischen Stoffen und Akteuren sichtbar werden.

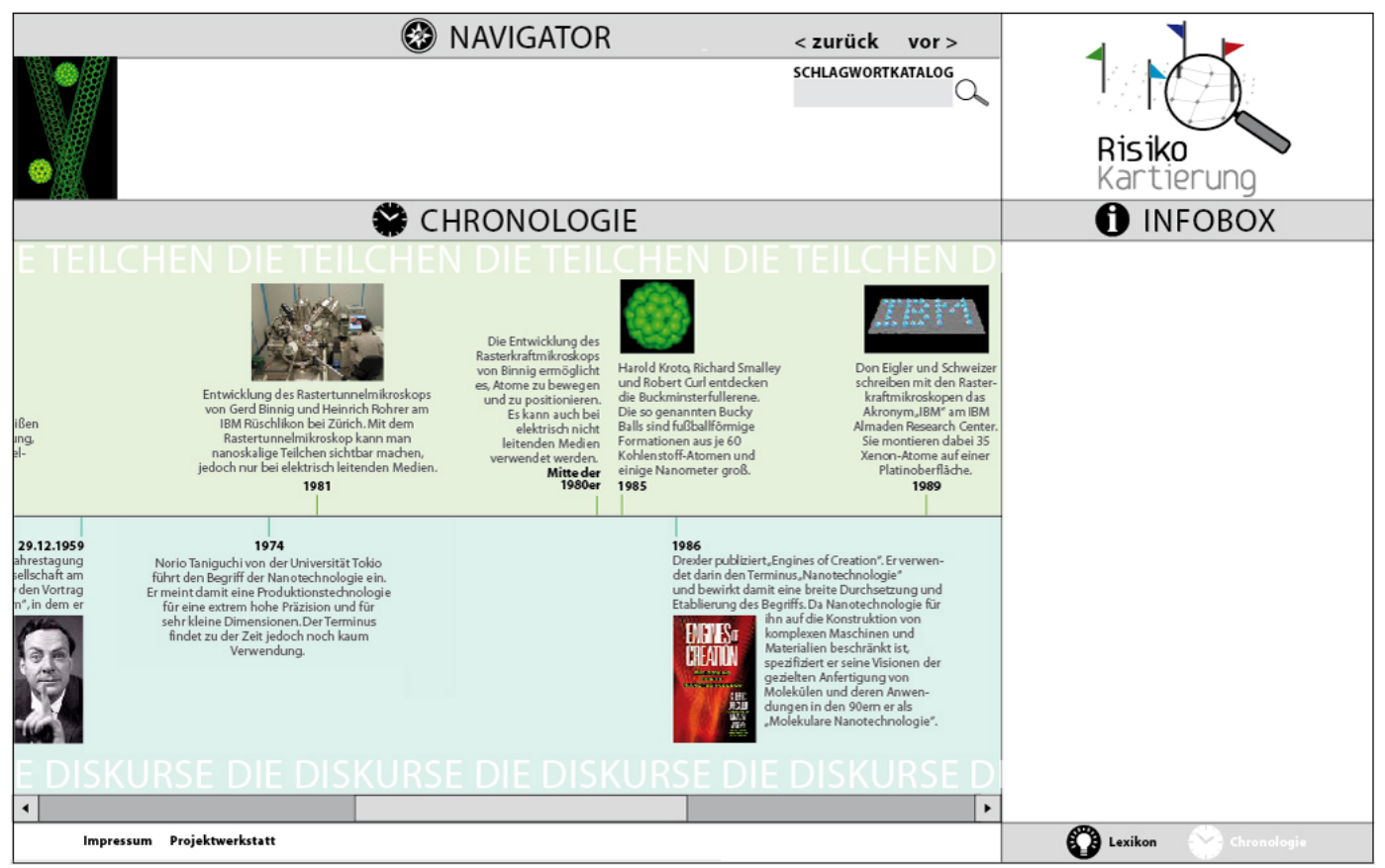

Abbildung 3: Visualisierungsentwurf: „,Chronologie der Nanopartikel-Debatte“ 


\section{Der Beitrag von kartierten Risikokonflikten zu Risikodebatten}

\section{I Leistungen und Herausforderungen}

Zusammenfassend sollen einige Potenziale und Herausforderungen skizziert werden, die sich im Hinblick auf die eingangs formulierten Ansprüche ergeben, die Kartierung als Instrument einer reflexiven Wissenspolitik zu entwickeln und einen Beitrag zu einem neuen, umfassenden Umgang mit systemischen Risiken zu leisten. Diesen wird das Werkzeug nicht nur durch die Vielfalt der abgebildeten Wissensbestände gerecht, sondern vor allem auch, weil hier die sozialwissenschaftliche Perspektive gestärkt und damit neue Sichtweisen ermöglicht werden.

Verstanden als Instrument der Risikokommunikation erweitert die Kartierung die bisher häufig stark naturwissenschaftlich-technische Perspektive um eine sozialwissenschaftliche insofern, als die debattierten Risiken gleichermaßen als Ausdruck von Diskursen und Netzwerken aufgefasst werden und dadurch die Wissensbasis, auf der es zu Entscheidungen über Risikotechnologien kommt, verbreitert wird. Dies ist eine der zentralen Leistungen des Instruments, da ein Einbeziehen der sozialwissenschaftlichen Perspektive Erkenntnisse über Zusammenhänge ermöglicht, die für erfolgreiche Risikoentscheidungen, also Entscheidungen, die von der Gesellschaft mitgetragen werden können, unerlässlich sind.

Ein weiterer Anspruch ist es, mit der Karte das „empowerment“ der breiten Öffentlichkeit in der Debatte um systemische Risiken zu fördern. Um den Bedürfnissen aller anvisierten Zielgruppen, zu denen sowohl VerbraucherInnen als auch Unternehmen, politische Entscheidungsträger, Nichtregierungsorganisationen und WissenschaftlerInnen gehören, gerecht zu werden, muss das Werkzeug eine relativ große Bandbreite sowohl im Hinblick auf die Inhalte als auch die Art der Darstellung aufweisen. Wie wird dies in der Karte umgesetzt? Die Strategie der Reduktion von Komplexität gewährleistet, dass die NutzerInnen relativ rasch einen Überblick über das Risikofeld und damit Zugang zur Debatte erhalten. Die Herausforderung besteht jedoch darin, die Balance zu finden zwischen nötiger Vereinfachung einerseits und einer gewissen Informations-Tiefe, die benötigt wird, um den Risikokonflikt erfassen und adäquat abbilden zu können andererseits. Gleichzeitig muss es für die NutzerInnen möglich sein, relativ einfach zwischen komplexeren und reduzierten Ansichten zu wechseln. Dies wird beispielsweise ermöglicht durch die unterschiedlichen Detailniveaus und den Wechsel zwischen „Komplettansicht“ und ,vereinfachter Ansicht“ (vgl. Darstellung der Streitfragenkomplexe oben). Den diversen Interessen potenzieller NutzerInnen wird außerdem durch die verschiedenen Einstiegsmöglichkeiten (über Akteure/Stoffe/Streitfragen) Rechnung getragen, ebenso durch das Angebot verschieden strukturierter Ansichten und unterschiedlicher Informationsebenen. Eine weitere Herausforderung besteht darin, die Balance zwischen bewusster Strukturierung, also einer stark interpretierenden Aufbereitung der Daten, und der Abbildung als „Sammlung von Vorgefundenem“, die eine stärkere Selbststeuerung durch die NutzerInnen ermöglicht, zu finden. Die kombinierte Darstellung von „Faktenwissen“, das unter anderem in der Info-Box oder im Lexikon enthalten ist und dessen Einbettung in diskursive Rahmungen oder Akteursnetzwerke bietet NutzerInnen zusätzliche Wahlmöglichkeiten. Die NutzerInnen können die unterschiedlichsten Perspektiven wählen, den Weg durch die Karte selbst entwickeln und haben damit die Möglichkeit einer relativ selbstbestimmten „Standortbestimmung“ zum jeweiligen Risiko. Dadurch, wird der in klassischen Settings häufig übliche Belehrungsgestus „Wissenschaft klärt auf - Bevölkerung hört zu“" zumindest partiell durchbrochen. 
Die angebotene Information ist angesichts der Fülle der sich im Diskurs zu Wort meldenden Akteure und der Vielfalt der Streitfragen, an denen sich Konflikte entzünden, notwendigerweise selektiv. Dies ist nicht nur aufgrund der Begrenzung durch den Bildschirm erforderlich, sondern auch, um Komplexitätsreduktion zu schaffen und damit Nachvollziehbarkeit zu gewährleisten. Jede Entscheidung über die Auswahl von Daten und die Art und Weise ihrer Abbildung stellt eine Interpretation dar. Es ist unabdingbar, das Selektionsverfahren den NutzerInnen gegenüber transparent zu machen und auf die Unvollständigkeit der dargebotenen Information hinzuweisen. Die Prinzipien zur Interpretation und Auswahl ergeben sich prinzipiell aus dem methodischen Zugang der Diskursanalyse, müssen im Detail jedoch noch ausgearbeitet werden. Klar ist jedoch, dass die zu kartierenden SprecherInnen so ausgewählt werden, dass der Risikokonflikt in seiner Differenziertheit erhalten bleibt. Weiterhin müssen gleiche Aussagen zusammengefasst werden. Wenn es darum geht im Sinne einer stärkeren Demokratisierung von Entscheidungsprozessen die Vielstimmigkeit des Diskurses zu abzubilden, ist es wichtig, auch die „leiseren“, weniger mächtigen Akteure aufzunehmen. Außerdem ist darauf zu achten, dass wissenschaftliches Wissen, Laienexpertise und kulturelles Erfahrungswissen gleichermaßen abgebildet werden.

Im Sinne einer social software als diskursives Forum werden NutzerInnen durch interaktive Elemente miteinbezogen, beispielsweise auf der „Werkstattseite“. Idealerweise ermöglicht ein „Gespräch“ zwischen KartiererInnen und NutzerInnen dieser Karten eine interaktive Stabilisierung der kartierten Diskursbilder. Allerdings müssen interaktive Prozesse geplant und gesteuert werden, um zu vermeiden, dass NutzerInnen, die über größere Ressourcen verfügen als andere, die Möglichkeiten der Interaktivitat besser nutzen können, um die Gestaltung der Kartierung zu beeinflussen und damit ihre spezifischen Interessen durchzusetzen. Dieses Problem betrifft jedoch nicht nur die Interaktivität: Bei allen Bemühungen, den unterschiedlichsten Interessen gerecht zu werden, wird es sich dennoch nicht vermeiden lassen, dass einzelne NutzerInnen unterschiedlich stark von den jeweils angebotenen Informationen profitieren. Beispielsweise können Unternehmen, die sich fragen, ob eine Marketingstrategie, die mit dem Begriff „Nanotechnologie“ operiert, beim Konsumenten greifen wird oder nicht, aus der Kartierung ersehen, ob Produkte, die Nanopartikel enthalten, eher positiv oder negativ konnotiert sind. Dies wird ersichtlich, wenn sie sich anschauen, welche Aussagen von Öffentlichkeitsakteuren wie Medien, Verbraucherverbänden, LaiInnen und BürgerInnen etc. über Nanopartikel eingebracht werden. Das gleiche gilt für PolitikerInnen, die sich zu ihrem eigenen Nutzen als Sprachrohr für die Sorgen der BürgerInnen in die Debatten einbringen wollen und dazu wissen wollen, welche Bedenken diese gegenüber Nanotechnologie oder auch Nahrungsergänzungsmitteln haben. Für diese Akteure fungiert die Karte quasi als Instrument der Meinungsforschung.

Die prinzipiell breite Zugänglichkeit ist durch das Internet zwar sichergestellt. Allerdings wirkt auch hier der Digital Divide. Denn nicht alle BürgerInnen haben Zugang zum Internet oder nutzen dieses bewusst als Informations- und Aktionsmedium. Da die Kartierung ein dynamisches Instrument darstellt, das sich mit den Debatten verändert, kann die Abbildung des Diskurses stets aktuell bleiben. Dies zu ermöglichen, setzt nicht nur entsprechende Weichenstellungen während der Entwicklungsphase voraus, sondern eine Pflege und Aufrechterhaltung der interaktiven Möglichkeiten über die Projektlaufzeit hinaus.

Elektronische Datenverarbeitung und Internetanwendungen ermöglichen nicht nur die Aufbereitung der Risikodiskurse, sondern auch die Diffusion von verschiedenen Wissensperspektiven, so dass die Kartierungen damit letztlich zu einer Strukturierung von Diskursen beitragen können. Auf diese Weise werden nicht nur die verschiedenen Risikoaspekte transparent - neben ökologischen und gesundheitlichen auch soziale Risiken wie etwa die Veränderung von Machtkonstellationen -, sondern gegebenenfalls können auch potenzielle, bisher nicht bekannte Risiken identifiziert oder Regulierungsbedarf deutlich werden. Nicht zuletzt zeigt sich in diesem Aspekt, dass die Kartierung neben der rekonstruierenden Rolle auch eine konstruierende übernimmt, denn sie selbst ist ebenfalls als Akteur in den Diskursen aufzufassen. 


\subsection{Ausblick}

In der Technikfolgenabschätzung (TA) ist mit dem Ruf nach einer demokratischen Basis für technologiepolitische Entscheidungen die Beteiligung von Betroffenen, BürgerInnen und WissenschaftlerInnen unterschiedlicher Disziplinen ein wichtiger Praxisbaustein geworden. Trotz der Beteiligung dieser Gruppen haftet einigen partizipativen Verfahren wie Risiko-Dialogen, Konsensus- und Verbraucherkonferenzen, Bürgerbeteiligungen und Mediationsverfahren eine expertenzentrierte Sicht der Dinge nach wie vor stärker an als es bei einem demokratieförderlichen Ansatz wünschenswert wäre (Joss und Bellucci 2002). Abschließend werfen wir daher die Frage auf, ob Risiko-Kartierungen wie die hier vorgestellte eine Antwort auf die vorgebrachte Kritik sein können und welchen Stellenwert derartige Kartierungen in der partizipativen TA haben können. Zum einen ist zu prüfen, in welcher Form eine Kartierung in einem Maßnahmenbündel zur partizipativen TA eingesetzt werden kann und ob sie etwa als vorbereitendes und ergänzendes Instrument für partizipative technologiepolitische Entscheidungsprozesse wie Konsensus-Konferenzen geeignet wäre. Zum anderen ist zu debattieren, ob und welche Vorteile eine Risiko-Kartierung gegenüber anderen partizipatorischen Maßnahmen bietet. Dazu möchten wir einige Aspekte in die Diskussion bringen:

- Maßnahmen der partizipativen TA werden in der Regel anlässlich akuter, kontrovers diskutierter Problemlagen angestoßen, bei denen dringender Entscheidungsbedarf besteht (Einsiedel et. al. 2001). Der Einbezug der Öffentlichkeit hat bei diesen Maßnahmen primär das Ziel, einen möglichst breit basierten Konsens herzustellen. Der Zwang zum Kompromiss bzw. zum Konsens hat der partizipativen TA bisweilen den Vorwurf eingebracht, es gehe auch bei den partizipativen Verfahren doch nur wieder darum, den Risikotechnologien gesellschaftliche Akzeptanz zu sichern. Eine Risikokartierung, die nach den oben beschriebenen Prinzipien angelegt wird, kann dieser Kritik teilweise standhalten, da sie keinem Entscheidungsbedarf Genüge tun muss und kein Zwang zum Konsens besteht, so dass dissensuelle Ansichten nicht in Form eines Kompromisses nivelliert werden müssen. Unterschiedliche Perspektiven auf die Gegenstände der Risikodebatten bleiben damit nicht nur erhalten, sondern sind in ihrer Differenziertheit sogar erwünscht. Fallweise können sie daher andere Formen der Auseinandersetzung über Risiken katalysieren.

- Der Einsatz von Risiko-Kartierungen als partizipatives Forum ist weder auf bestimmte Zeitabschnitte begrenzt noch auf bestimmte Örtlichkeiten beschränkt. Dadurch können breitere Kreise von NutzerInnen erschlossen werden.

- Die über die interaktiven Elemente der Kartierung am Diskurs teilnehmenden Akteure werden nicht von etwaigen Initiatoren eines partizipativen Instrumentes vorab ausgewählt, wie es bei der Vorbereitung eines Panels für eine Konsensus-Konferenz oder eines Mediationsverfahrens unvermeidbar ist. Dies verbreitert die demokratische Basis um weitere Gruppen von NutzerInnen zusätzlich.

Die aufgezählten Aspekte konnten in diesem Rahmen nur angerissen und nicht detailliert diskutiert werden. Sie mögen aber zur vertieften Auseinandersetzung anregen. 


\section{Literaturverzeichnis}

Abels, G., Bora, A., 2004, Demokratische Technikbewertung, Bielefeld: transcript.

aid Infodienst Verbraucherschutz, Ernährung, Landwirtschaft e.V., 2005, Nahrungsergänzungsmittel - Nutzen oder Risiko?, Bonn: aid Infodienst e.V.

Beck, U., 1986, Risikogesellschaft, Auf dem Weg in eine andere Moderne, Frankfurt (M.): Suhrkamp.

BMVEL (Bundesministerium für Verbraucherschutz, Ernährung und Landwirtschaft), (Hg.), 2004 : Verordnung über Nahrungsergänzungsmittel und zur Änderung der Verordnung über vitaminisierte Lebensmittel. Bundesgesetzblatt Teil I Nr. 25 vom 27. Mai 2004, 1011-1015

Böschen, S., 2004, Science Assessment: Eine Perspektive der Demokratisierung von Wissenschaft, in: Böschen, S. und Wehling, P. (Hg.): Wissenschaft zwischen Folgenverantwortung und Nichtwissen. Aktuelle Perspektiven der Wissenschaftsforschung, Wiesbaden: VS Verlag für Sozialwissenschaften, 107-182.

Böschen, S., 2005, Reflexive Wissenspolitik. Formierung und Strukturierung von Gestaltungsöffentlichkeiten, in: Bogner, A. und Torgersen, H. (Hg.): Wozu Experten? Ambivalenzen der Beziehung von Wissenschaft und Politik, Wiesbaden: VS Verlag für Sozialwissenschaften, 241-263.

Böschen, S., Wehling, P., 2004, Wissenschaft zwischen Folgenverantwortung und Nichtwissen. Aktuelle Perspektiven der Wissenschaftsforschung, Wiesbaden: VS Verlag für Sozialwissenschaften.

Bonß, W., 1991, Unsicherheit und Gesellschaft - Argumente für eine soziologische Risikoforschung, Soziale Welt 42(2), 258-277.

Bonß, W., 1995, Vom Risiko: Unsicherheit und Ungewissheit in der Moderne, Hamburg: Hamburger Edition.

Callon, M., 1999, Some Elements of a sociology of Translation. Domestication of the Scallops and the Fishermen of St. Brieuc Bay, in: Biagioli, M. (Hg.): The Science Studies Reader, 67-83.

Diedrich, H., 2002, Nahrungsergänzungsmittel: Sinn und Unsinn beim Einsatz im Sport, Köln: Verlag Sport und Buch Strauß.

Einsiedel, E., Jelsøe, E., Breck, T., 2001, Publics at the Technology Table: The Consensus Conference in Denmark, Canada, and Australia, Public Understanding of Science 10(1), 83-89.

Epstein, S., 1995, The Construction of Lay Expertise: AIDS Activism and the Forging of Credibility in the Reform of Clinical Trials, Science, Technology \& Human Values 20(4), 408-437.

Felt, U., 2002, Sciences, Science Studies and their Publics: Speculations on Future Relations, in: Nowotny, H. und Joerges, B. (Hg.), Social Studies of Science \& Technology: Looking back ahead, Yearbook of the Sociology of the Sciences, 11-31.

Felt, U., 2003, Scientific Citizenship. Schlaglichter einer Diskussion, Gegenworte. Hefte für den Disput über Wissen 11, 16-20.

Grove-White, R., Kearnes, M., Miller, P., Wynne, B., Macnaghten, P. and Wilsdon, J., 2004, Bio to - Nano? Learning the Lessons, Interrogating the Comparison, Working Paper by the Institute for Environment, Philosophy and Public Policy, Lancaster: Lancaster University and Demos. 
Hahn, A., Wolters, M., Hülsmann, O., 2006, Nahrungsergänzungsmittel und ergänzende bilanzierte Diäten, Stuttgart: Wissenschaftliche Verlagsgesellschaft mbH.

Irwin, A., 1995, Citizen Science. A Study of People, Expertise and Sustainable Development, New York: Routlege.

Irwin, A., Wynne, B., 1996, Misunderstanding science? The public reconstruction of science and technology, Cambridge: Cambridge University Press.

Jäger, S., 2001, Kritische Diskursanalyse, Duisburg: Duisburger Institut für Sprach- und Sozialforschung.

Jasanoff, S., 2003, Breaking the Waves in Science Studies, Social Studies of Science 33(3), 389400 .

Joss, S., Bellucci, S., 2002, Participatory Technology Assessment: European Perspectives, London: Westminster University Press.

Keller, R., Hirseland, A., Schneider, W., Viehöver,W. (Hg.), 2001, Handbuch Sozialwissenschaftliche Diskursanalyse: Theoretische und methodische Grundlagen (Band 1), Opladen: Leske+ Budrich.

Keller, R., Hirseland, A., Schneider, W., Viehöver,W. (Hg.), 2003, Handbuch Sozialwissenschaftliche Diskursanalyse: Forschungspraxis (Band 2), Opladen: Leske+Budrich.

Klinke, A., Renn, O., 2006, Systemic Risks as Challenge for Policy Making in Risk Governance, in: Forum Qualitative Sozialforschung/Forum: Qualitative Social Research, 7(1), Art. 33, (adopted on 14 february, 2007)

<http://www.qualitativeresearch.net/fqs-texte/I-06/06-I-33-e.htm>.

Krohn, W., Krücken, G., 1993, Risiko als Konstruktion und Wirklichkeit. Eine Einführung in die sozialwissenschaftliche Risikoforschung, in: Dies. (Hg.): Riskante Technologien: Reflexion und Regulation, Frankfurt (M.): Suhrkamp, 9-44.

Knorr-Cetina, K., 1999, Epistemic Cultures, Cambridge, MA: Harvard University Press.

Latour, B., 1983, Give me a laboratory and I will raise the world, in: Knorr-Cetina, K. und Mulkay, M.: Science observed. London: Sage.

Latour, B., 2006, Von der ,wissenschaftlichen Wahrheit” zur Kartographie von Kontroversen, in: Liebert, W.A. und Weitze, M.D. (Hg.): Kontroversen als Schlüssel zur Wissenschaft? Wissenskulturen in sprachlicher Interaktion, Bielefeld: transcript Verlag, 195-202.

Lau, C., 1989, Risikodiskurse: Gesellschaftliche Auseinandersetzungen um die Definition von Risiken, Soziale Welt 40(3), 418-436.

Lévy-Leblond, J.-M., 1992, About misunderstandings about misunderstandings, Public Understanding of Science 1(1), 17-21.

Limoges, C., 1993, Expert Knowledge and Decision-Making in controversy contexts, Public Understanding of Science 2(4), 417-426.

Nowotny, H., 1999, The Need for Socially Robust Knowledge, TA-Datenbank-Nachrichten 8 (3/4), 12-16.

OECD (Organisation for Economic Co-operation and Development), 2003a, Emerging Systemic Risks in the $21^{\text {st }}$ Century: An Agenda for Action (Overview), Paris: OECD.

OECD (Organisation for Economic Co-operation and Development), 2003b, Kurzfassung - Neue Risiken im 21. Jahrhundert: Vorschläge für ein Aktionsprogramm, Paris: OECD. 
Paine, R., 1992, Chernobyl reaches Norway: the Accident, Science and the Threat to Cultural Knowledge, Public Understanding of Science 1(3), 261-280.

Parr, D., 2005, Will nanotechnology make the world a better place? in: Nanoforum (Hg.), Benefits, Risks, Ethical, Legal and Social Aspects of Nanotechnology, (adopted on 11 january 2006) http://www.nanoforum.org.

Perrow, C., 1984, Normale Katastrophen. Die unvermeidbaren Risiken der Großtechnik, Frankfurt (M.): Suhrkamp.

Rogers, R., Marres, N., 2001, Landscaping climate change: a mapping technique for understanding science and technology debates on the World Wide Web, Public Understanding of Science 9, $1-12$.

Rogers, R., 2004, Information Politics on the Web. Harvard/Massachusetts: MIT Press.

Stehr, N., 2003, Wissenspolitik. Die Überwachung des Wissens, Frankfurt (M.): Suhrkamp.

Swiss Re, 2004, Nanotechnology. Small Matter - many unknowns, Reihe Risk Perception, Zürich: Swiss Reinsurance Company.

Verbraucherzentrale Bundesverband e.V., 2005, Nahrungsergänzungsmittel. Gesünder leben durch Pillen und Pulver? Berlin: Verbraucherzentrale Bundesverband.

WBGU (Wissenschaftlicher Beirat der Bundesregierung Globale Umweltveränderungen), 1999, Welt im Wandel: Strategien zur Bewältigung globaler Umweltrisiken. Jahresgutachten 1998, Berlin: Springer.

Wehling, P., 2004a, Reflexive Wissenspolitik: Öffnung und Erweiterung eines neuen Politikfeldes, Technikfolgenabschätzung - Theorie und Praxis13(3), 63-71.

Wehling, P., 2004b, Weshalb weiß die Wissenschaft nicht, was sie nicht weiß? Umrisse einer Soziologie des wissenschaftlichen Nichtwissens, in: Böschen, S. und Wehling, P. (Hg.): Wissenschaft zwischen Folgenverantwortung und Nichtwissen. Aktuelle Perspektiven der Wissenschaftsforschung, Wiesbaden: VS Verlag für Sozialwissenschaften, 35-105.

Weingart, P., 2003, Wissenschaftssoziologie, Bielefeld: transcript.

Wynne, B., 1992, Misunderstood misunderstandings: Social Identities and the public uptake of science, Public Understanding of Science 1(3), 281-304.

Wynne, B., 1993, Public uptake of Science: a case for institutional reflexivity, Public Understanding of Science 2(4), 321-337.

Wynne, B., 1995, Public Understanding of Science, in: Jasanoff, S., Markle, G. E., Peterson, J. C. and Pinch, T. (Hg.): Handbook of Science and Technology Studies, Thousands Oaks: Sage, 361-388.

Wynne, B., 1996, May the sheep safely graze? A reflexive view of the expert-lay knowledge divide, in: Lash, S., Szerszynski, B. und Wynne, B. (Hg.): Risk, Environment and Modernity. Towards a New Ecology, London: Sage, 44-83. 


\section{Bisher erschienene manu:scripte}

ITA-0I-0I Gunther Tichy, Walter Peissl (12/2001): Beeinträchtigung der Privatsphäre in der Informationsgesellschaft. <http://www.oeaw.ac.at/ita/pdf/ita_0I_0I.pdf>

ITA-0I-02 Georg Aichholzer(12/2001): Delphi Austria: An Example of Tailoring Foresight to the Needs of a Small Country. <http://www.oeaw.ac.at/ita/pdf/ita_0I_02.pdf>

ITA-0I-03 Helge Torgersen, Jürgen Hampel (12/2001): The Gate-Resonance Model: The Interface of Policy, Media and the Public in Technology Conflicts.

<http://www.oeaw.ac.at/ita/pdf/ita_0l_03.pdf>

ITA-02-0I Georg Aichholzer (01/2002): Das ExpertInnen-Delphi: Methodische Grundlagen und Anwendungsfeld ,Technology Foresight“. <http://www.oeaw.ac.at/ita/pdf/ita_02_0I.pdf>

ITA-02-02 Walter Peissl (01/2002): Surveillance and Security - A Dodgy Relationship. $<$ http://www.oeaw.ac.at/ita/pdf/ita_02_02.pdf $>$

ITA-02-03 Gunther Tichy (02/2002): Informationsgesellschaft und flexiblere Arbeitsmärkte. $<$ http://www.oeaw.ac.at/ita/pdf/ita_02_03.pdf >

ITA-02-04 Andreas Diekmann (06/2002): Diagnose von Fehlerquellen und methodische Qualität in der sozialwissenschaftlichen Forschung. <http://www.oeaw.ac.at/ita/pdf/ita_02_04.pdf>

ITA-02-05 Gunther Tichy (10/2002): Over-optimism Among Experts in Assessment and Foresight. <http://www.oeaw.ac.at/ita/pdf/ita_02_05.pdf>

ITA-02-06 Hilmar Westholm (12/2002): Mit eDemocracy zu deliberativer Politik? Zur Praxis und Anschlussfähigkeit eines neuen Mediums. <http://www.oeaw.ac.at/ita/pdf/ita_02_06.pdf>

ITA-03-0I Jörg Flecker und Sabine Kirschenhofer (01/2003): IT verleiht Flügel? Aktuelle Tendenzen der räumlichen Verlagerung von Arbeit. $<$ http://www.oeaw.ac.at/ita/pdf/ita_03_0l.pdf>

ITA-03-02 Gunther Tichy (11/2003): Die Risikogesellschaft - Ein vernachlässigtes Konzept in der europäischen Stagnationsdiskussion.

$<$ http://www.oeaw.ac.at/ita/pdf/ita_03_02.pdf>

ITA-03-03 Michael Nentwich (11/2003): Neue Kommunikationstechnologien und Wissenschaft - Veränderungspotentiale und Handlungsoptionen auf dem Weg zur Cyber-Wissenschaft. <http://www.oeaw.ac.at/ita/pdf/ita_03_03.pdf>

ITA-04-0I Gerd Schienstock (1/2004): Finnland auf dem Weg zur Wissensökonomie - Von Pfadabhängigkeit zu Pfadentwicklung. <http://www.oeaw.ac.at/ita/pdf/ita_04_0I.pdf>

ITA-04-02 Gunther Tichy (6/2004): Technikfolgen-Abschätzung: Entscheidungshilfe in einer komplexen Welt. <http://www.oeaw.ac.at/ita/pdf/ita_04_02.pdf>

ITA-04-03 Johannes M. Bauer (11/2004): Governing the Networks of the Information Society - Prospects and limits of policy in a complex technical system.

<http://www.oeaw.ac.at/ita/pdf/ita_04_03.pdf>

ITA-04-04 Ronald Leenes (12/2004): Local e-Government in the Netherlands: From Ambitious Policy Goals to Harsh Reality. <http://www.oeaw.ac.at/ita/pdf/ita_04_04.pdf>

ITA-05-0I Andreas Krisch (01/2005): Die Veröffentlichung des Privaten - Mit intelligenten Etiketten vom grundsätzlichen Schutz der Privatsphäre zum Selbstschutz-Prinzip. <http://www.oeaw.ac.at/ita/pdf/ita_05_0I.pdf> 
ITA-05-02 Petra Grabner (12/2005): Ein Subsidiaritätstest - Die Errichtung gentechnikfreier Regionen in Österreich zwischen Anspruch und Wirklichkeit.

<http://www.oeaw.ac.at/ita/pdf/ita_05_02.pdf>

ITA-05-03 Eva Buchinger (12/2005): Innovationspolitik aus systemtheoretischer Sicht - Ein zyklisches Modell der politischen Steuerung technologischer Innovation. $<$ http://www.oeaw.ac.at/ita/pdf/ita_05_03.pdf>

ITA-06-0I Michael Latzer (06/2006): Medien- und Telekommunikationspolitik: Unordnung durch Konvergenz - Ordnung durch Mediamatikpolitik. $<$ http://epub.oeaw.ac.at/ita/ita-manuscript/ita_06_0I.pdf $>$

ITA-06-02 Natascha Just, Michael Latzer, Florian Saurwein (09/2006): Communications Governance: Entscheidungshilfe für die Wahl des Regulierungsarrangements am Beispiel Spam. <http://epub.oeaw.ac.at/ita/ita-manuscript/ita_06_02.pdf >

ITA-06-03 Veronika Gaube, Helmut Haberl (10/2006): Sozial-ökologische Konzepte, Modelle und Indikatoren nachhaltiger Entwicklung: Trends im Ressourcenverbrauch in Österreich. $<$ http://epub.oeaw.ac.at/ita/ita-manuscript/ita_06_03.pdf>

ITA-06-04 Maximilian Fochler, Annina Müller (11/2006): Vom Defizit zum Dialog? Zum Verhältnis von Wissenschaft und Öffentlichkeit in der europäischen und österreichischen Forschungspolitik.

$<$ http://epub.oeaw.ac.at/ita/ita-manuscript/ita 06 04.pdf>

ITA-06-05 Holger Floeting (11/2006): Sicherheitstechnologien und neue urbane Sicherheitsregimes. $<$ http://epub.oeaw.ac.at/ita/ita-manuscript/ita_06_05.pdf>

ITA-06-06 Armin Spök (12/2006): From Farming to "Pharming" - Risks and Policy Challenges of Third Generation GM Crops. <http://epub.oeaw.ac.at/ita/ita-manuscript/ita_06_06.pdf>

ITA-07-0I Volker Stelzer, Christine Rösch, Konrad Raab (3/2007): Ein integratives Konzept zur Messung von Nachhaltigkeit - das Beispiel Energiegewinnung aus Grünland. $<$ http://epub.oeaw.ac.at/ita/ita-manuscript/ita_07_0I.pdf $>$

ITA-07-02 Elisabeth Katzlinger (3/2007): Big Brother beim Lernen: Privatsphäre und Datenschutz in Lernplattformen.

$<$ http://epub.oeaw.ac.at/ita/ita-manuscript/ita_07_02.pdf $>$

ITA-07-03 Astrid Engel, Martina Erlemann (4/2007): Kartierte Risikokonflikte als Instrument reflexiver Wissenspolitik. <http://epub.oeaw.ac.at/ita/ita-manuscript/ita_07_03.pdf> 